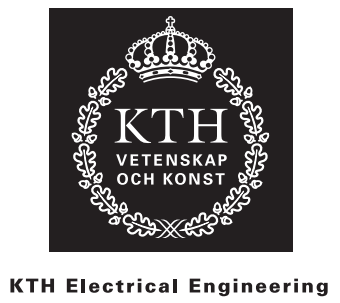

\title{
A Multichoice Control Strategy for a VSC-HVdc
}

HÉCTOR F. LATORRE S.

Licentiate Thesis

Royal Institute of Technology

School of Electrical Engineering

Electric Power Systems

Stockholm, Sweden, 2008 
TRITA-EE 2008:09

ISSN $1653-5146$

ISBN 978-91-7178-880-1
School of Electrical Engineering Electric Power Systems Royal Institute of Technology SE-100 44 Stockholm Sweden

Akademisk avhandling som med tillstånd av Kungl Tekniska högskolan framlägges till offentlig granskning för avläggande av teknologie licentiatexamen måndagen den 07 april 2008 kl 10.00 i V32, Teknikringen 72, Kungl Tekniska Högskolan, Stockholm.

(c) Héctor F. Latorre S., March 2008

Tryck: Universitetsservice US-AB 


\begin{abstract}
Utilization of power electronics based controllable systems (or devices) in transmission systems has opened new opportunities for the power industry to optimize utilization of the existing transmission systems, and at the same time to keep high system reliability and security

As a member of these controllable systems, Voltage Source Convertersbased High Voltage direct current (VSC-HVdc) systems have the ability to rapidly control the transmitted active power, and also to independently exchange reactive power with transmissions systems. Therefore, VSC-HVdcs with a suitable control scheme can offer an alternative means to enhance transient stability, to improve power oscillations damping, and to provide voltage support. An interesting application of this system is the analysis of a power system when a VSC-HVdc is connected in parallel with ac transmission lines.

This thesis presents the derivation of control strategies to damp power oscillations, to enhance the transient stability and to provide voltage support for a VSC-HVdc. The thesis also formulates a multichoice control strategy and its application when the VSC-HVdc is connected in a synchronous system.

The control strategy for enhancing transient stability is based on the theory of Control Lyapunov Function. The control strategy for increasing the damping is based on Linear Analysis. A very effective well known way to increase damping in the system is modulating the active power through the HVdc. However, besides the control of active power, the thesis explores an alternative way to mitigate power oscillations by controlling the reactive power. This condition might be very useful when the dc link in the VSCHVdc system is out of service, but the converter stations are in operating conditions.
\end{abstract}

A simple model of VSC-HVdc is considered in order to test the control 
strategy. The model represents the VSC-HVdc as an element in the power system that provides adequate interaction with other systems elements. The model is intended for analysis of power flows and electromechanical transients. It is then sufficient to consider the power frequency components of voltages and currents represented by phasors that vary with time during transients. The model is valid for symmetrical conditions, i.e. positive sequence phasors are used for the representation of the electrical state.

Keywords. Control Lyapunov Function, Modal Analysis, Power Oscillation Damping, Transient Stability, Voltage Stability, VSC-HVdc. 


\section{Acknowledgment}

The financial support of the project from ELEKTRA program is gratefully acknowledged.

I would like to thank Professor Lennart Söder for letting me know about the open position for this PhD program. I also thank him for his discussions, comments, suggestions and feedback during the research.

I would like to thank my supervisor, Assistant Professor, Mehrdad Ghandhari for his endless patient, his guidance during the research, his extensive theoretical explanations and his help with my simulations in SIMPOW. I especially thank him all the time he dedicated to read the papers and Licentiate Thesis.

Many thanks to ABB HVDC for providing KTH with its model of the HVDC-Light (C) in order to be used in the simulations of this project.

I also want to thank all the members of the reference group for very interesting meetings, helpful discussions and meaningful comments about the partial results and future steps during the project.

I extend my personal thanks to the staff of Electric Power Systems for a friendly environment to study and research. Especially thanks to Margaretha Surjadi for all her assistance.

I would like to thank my friends Juan Carlos, Rigoberto and Gloria for his love and care.

Finally, my deepest gratitude to my parents, niece, sister and brother for all their love and support; their endless care despite of the near $10.000 \mathrm{~km}$ of distance. Rubén and Mariela, my parents, thanks for teaching me the precious value of education. 



\section{Contents}

Contents vii

$\begin{array}{ll}\text { List of Figures } & \text { ix }\end{array}$

List of Symbols $\quad$ xi

1 Introduction $\quad 1$

1.1 Current Challenges in Power Systems . . . . . . . . . . . 1

1.2 VSC-HVdc on Power Systems . . . . . . . . . . . 2

1.3 Why VSC-HVdc . . . . . . . . . . . . . . 2

1.4 Applied Methods in the Control Strategies . . . . . . . . . . 4

1.5 Multichoice Control Strategy . . . . . . . . . . . . . 4

1.6 Objectives . . . . . . . . . . . . . . . 5

1.7 Outline of the Thesis . . . . . . . . . . . . 6

1.8 Main contributions . . . . . . . . . . . . 7

1.9 List of Publications . . . . . . . . . . . . . 8

2 Modeling of VSC-HVdc 9

2.1 VSC-HVdc . . . . . . . . . . . . . . . . . . 9

2.2 Injection Model of VSC-HVdc . . . . . . . . . . . . 12

3 Theoretical Background $\quad 17$

3.1 Power System Modeling . . . . . . . . . . . . . . 17

3.1.1 Synchronous Machines . . . . . . . . . . . . 18

3.1 .2 Static Load . . . . . . . . . . . . . . . . 20

3.1.3 Multi-Machine Power System . . . . . . . . . . . 22

3.2 Lyapunov Theory . . . . . . . . . . . . . . . . . . . 24

vii 
3.3 Application of Lyapunov Theory to Multi-Machine Power Systems ........................ . . 30

3.4 Control Lyapunov Function (CLF) . . . . . . . . . . . . 35

3.5 Modal Analysis . . . . . . . . . . . . . . . . . 37

3.5.1 Tuning of Regulator ................ . 41

4 Control Strategies for a VSC-HVdc $\quad 45$

4.1 Control Lyapunov Function . . . . . . . . . . . . . . 45

4.2 Modal Analysis . . . . . . . . . . . . . . . . . . . . 49

4.2.1 Active Power Control . . . . . . . . . . . . . . . . 50

4.2.2 Reactive Power Control . . . . . . . . . . . . . . . 50

4.2.3 Modulative Part of the Controllable Variables . . . . . 51

4.3 Multichoice Control Strategy . . . . . . . . . . . . . 52

$5 \quad$ Numerical Examples $\mathbf{5 3}$

5.1 Test Power System . . . . . . . . . . . . . . . . . . . 53

5.2 Overall Control of the VSC-HVdc . . . . . . . . . . . . 55

5.3 Comparison of VSC-HVdc Models. Active Power Modulation and AVR ....................... 56

5.4 Only Active Power Modulation . . . . . . . . . . . . 58

5.4.1 Active Power Modulation and AVR . . . . . . . . . . . 60

5.5 Combination of CLF-Based Control Strategy and ModalAnalysis-Based Control Strategy ........... . 64

5.6 Reactive Power Control .............. . 65

5.7 Power Flow Control . . . . . . . . . . . . . . 68

6 Conclusions and Future Work $\quad 75$

6.1 Conclusions ..................... . . . 75

6.2 Future Work .................. . . 77

$\begin{array}{lr}\text { Bibliography } & 79\end{array}$ 


\section{List of Figures}

1.1 Multichoice Control . . . . . . . . . . . . . . . 5

2.1 Simplified single line diagram for VSC-HVdc . . . . . . . . . . 10

$2.2 \mathrm{P}$ - Q diagram for the whole dc voltage range . . . . . . . . . 11

2.3 Basic Structure of VSC-HVdc. . . . . . . . . . . . . . 12

2.4 Simple model of a VSC-HVdc. . . . . . . . . . . . . . . . 13

2.5 Injection model of a VSC-HVdc. . . . . . . . . . . . . 14

3.1 Synchronous generator one-axis dynamic circuit . . . . . . . . 23

3.2 SMIB system . . . . . . . . . . . . . . . . . . . . . . . . . . 29

3.3 Feedback control system . . . . . . . . . . . . . . . . . 41

3.4 Damping Regulator . . . . . . . . . . . . . . . . . . . . 41

3.5 Direction of the eigenvalue departure . . . . . . . . . 42

4.1 Active power control . . . . . . . . . . . . . . . 50

4.2 Reactive power control . . . . . . . . . . . . . . . 51

4.3 Multichoice Control Strategy . . . . . . . . . . . . . . 52

5.1 Test Power System . . . . . . . . . . . . . . . . . . . 53

5.2 Proposed Scheme Control of the VSC-HVdc . . . . . . . . . 55

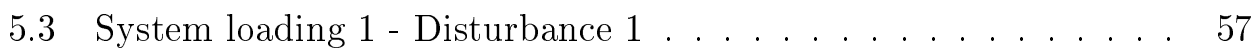

5.4 System loading 1 - Disturbance $3 \ldots \ldots \ldots . . \ldots 57$

5.5 System loading 3 - Disturbance $3 \ldots \ldots \ldots . \ldots 58$

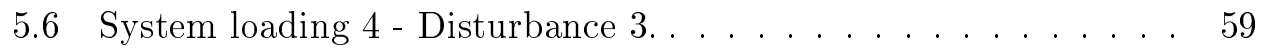

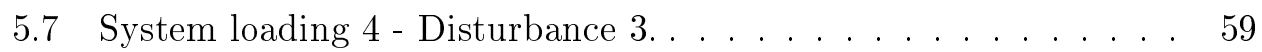

5.8 System loading 4 - Disturbance $3 \ldots \ldots \ldots 61$

5.9 System loading 4 - Disturbance $3 \ldots \ldots \ldots 1$

5.10 System loading 4 - Disturbance 1. . . . . . . . . . . . . 62 
5.11 System loading 1 - Disturbance 4. . . . . . . . . . . . 63

5.12 System loading 1 - Disturbance 4. . . . . . . . . . . . 63

5.13 System loading 1 - Disturbance 4. . . . . . . . . . . . . 64

5.14 System loading 4 - Disturbance 3. . . . . . . . . . . 65

5.15 System loading 4 - Disturbance 3. . . . . . . . . . . 66

5.16 System loading 2 - Disturbance 6 . . . . . . . . . . 67

5.17 System loading 2 - Disturbance 6 . . . . . . . . . . . 67

5.18 System response to manual change of $P_{\text {sio }} \ldots$. . . . . . . . . . 69

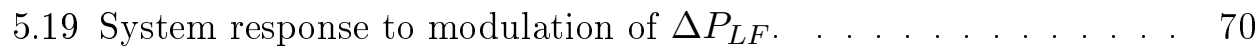

5.20 System response to disturbance 2 . . . . . . . . . . . . . 71

5.21 System loading 4 - Disturbance 2. . . . . . . . . . . . . 72

5.22 System loading 4 - Disturbance 2. . . . . . . . . . . . 72

5.23 System loading 4 - Disturbance $2 \ldots . . . . . . . . . .73$ 


\section{List of Symbols}

$E_{i} \quad$ Magnitude of the controllable voltage source at end $i$

$E_{j} \quad$ Magnitude of the controllable voltage source at end $j$

$E_{i, j}^{i m} \quad$ Imaginary part of the controllable voltage source

$E_{i, j}^{r e} \quad$ Real part of the controllable voltage source

$E_{i o, j o}^{i m} \quad$ Fixed variable (or set value) of $E_{i, j}^{i m}$

$E_{i o, j o}^{r e} \quad$ Fixed variable (or set value) of $E_{i, j}^{r e}$

$P_{s i, s j} \quad$ Active power injected by the VSC-HVdc into the ac network at bus $i, j$

$P_{\text {sio,sjo }} \quad$ Uncontrolled active power injected into the VSC-HVdc from the ac network at bus $i, j$

$Q_{s i, s j} \quad$ Reactive power injected by the VSC-HVdc into the ac network at bus $i, j$

$Q_{\text {sio,sjo }} \quad$ Uncontrolled reactive power injected into the VSC-HVdc from the ac network at bus $i, j$

$U_{i, j} \quad$ Magnitude of the ac voltage at bus $i, j$

$\Delta E_{i o, j o}^{i m} \quad$ Modulated variable of $E_{i, j}^{i m}$

$\Delta E_{i o, j o}^{r e} \quad$ Modulated variable of $E_{i, j}^{r e}$

$\Delta P_{s i, s j} \quad$ Controlled active power injected into the VSC-HVdc from the ac network at bus $i, j$ 
$\Delta Q_{s i, s j} \quad$ Controlled reactive power injected into the VSC-HVdc from the ac network at bus $i, j$

$\gamma_{i}$

$\gamma_{j}$

$\theta_{i, j}$

$E_{q}^{\prime}$

M

$P_{e}$

$P_{m}$

$P_{g k}$

$P_{k}$

$Q_{g k}$

$Q_{k}$

$\delta$

$\omega$

$x_{d}^{\prime}$

$\delta_{C O I}$

$\dot{\mathcal{V}}(x)$

$\lambda_{i}$

$\mathcal{V}(x)$

$\omega_{C O I}$

$\tilde{\delta}_{k}$

$\tilde{\omega}_{k}$
Phase angle of the controllable voltage source at end $i$

Phase angle of the controllable voltage source at end $j$

Angle of the ac voltage at bus $i, j$

$q$-axis transient emf

Inertia constant

Electrical power

Mechanical power

Active power supplied by the generator $k$

Active power injected into bus $k$

Reactive power supplied by the generator $k$

Reactive power injected into bus $k$

Angular position of the rotor relative to a reference synchronous machine with synchronous speed

Rotor speed

$d$-axis transient reactance

Rotor angle of the Center Of Inertia

Time derivative of $\mathcal{V}(x)$ along the trajectories of a system

Eigenvalue of the mode $i$

Lyapunov function (or energy function)

Rotor speed of the Center Of Inertia

Rotor angle of generator $k$ in the COI reference frame

Rotor speed of generator $k$ in the COI reference frame 
$\tilde{\theta}_{k} \quad$ Phase angle of bus $k$ of the transmission network in the COI reference frame

A State matrix

$B \quad$ Input matrix

C Output matrix

D $\quad$ Feedforward matrix

$G(s) \quad$ Transfer function of the uncoupled differential algebraic system

$R_{i} \quad$ Residue of $G(s)$ at $\lambda_{i}$

$T_{1}, T_{2} \quad$ Time constants of the lead-lag filter

$T_{w} \quad$ Time constant of the wash-out filter

$V^{L} \quad$ Matrix of left eigenvectors

$V^{R} \quad$ Matrix of right eigenvectors

$\Lambda \quad$ Diagonal matrix of eigenvalues

$\alpha_{\max } \quad$ Maximum phase lead angle

$\mathcal{Y} \quad$ Output vector

$\omega_{\max } \quad$ Maximum frequency at $\alpha_{\max }$

$\phi \quad$ Argument of the transfer function of the regulator

$\xi \quad$ State vector of the uncoupled differential algebraic system

$u \quad$ Input vector

$x \quad$ State variables vector

$\mathcal{V}_{H V d c_{o}} \quad$ Part of the energy function of the uncontrolled system corresponding to the uncontrolled VSC-HVdc

$\mathcal{V}_{\text {uncontrolled }}$ Energy function of the uncontrolled system 


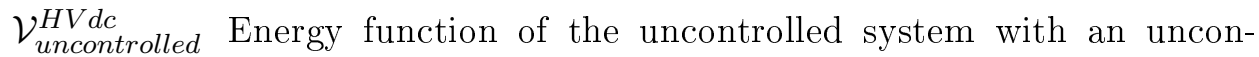
trolled VSC-HVdc

Iline $\quad$ Magnitude of a line current used as input signal in the active and reactive power control

$\Delta f \quad$ Difference of frequencies measured at two buses in the network used as input signal in the active and reactive power control 


\section{Chapter 1}

\section{Introduction}

\subsection{Current Challenges in Power Systems}

Traditionally, one common way to improve the level of reliability, through scheduled interchange of power between different areas, is the interconnection of transmission systems. Formerly separated systems are connected to each other evolving into power systems of very large size, expanding hundreds and thousands of kilometers, steadily growing with ever larger capacity. However, in present scenario of electricity supply business, these interconnections are used for trading even though they were not designed for this purpose. Long distance power trading puts more stress on the existing transmission system. As a result, low frequency oscillations involving the poorly damped inter-area modes become more pronounced risking the system security [1].

Low frequency electromechanical oscillations are inherent in large interconnected power systems. These oscillations, commonly referred to as "inter-area oscillations", are associated with groups of synchronous generators in one geographical region swinging with respect to other groups in a different region interconnected through tie-lines. Adequate damping of the oscillations is a pre-requisite for secure operation of the system [1]. Over the years, many incidents of system outage resulting from these oscillations have been reported. One example is the August 10, 1996 blackout in the WSCC system of United States [2].

Today, the average transmission line is loaded more heavily than ever before and this has given rise to a new problem, voltage instability [3]. The voltage collapse usually occurs following a large disturbance (such as a fault 
or load increase) in a heavily stressed power system, which results in increased reactive power consumption and hence leads to a voltage drop. The voltage drop leads to initial load reduction bringing load restoration control mechanisms into action. It is the dynamics of these controls that often lead to voltage instability and collapse [4]. Some examples are the blackout in USA and Canada in August 14, 2003 [5]; the blackout in Sweden and Denmark in September 23, 2003; or the blackout in south Greece in July 12, 2004 [6].

Power systems must offer a good level of flexibility, controllability and reliability to securely react under sudden changes in the system due to either alterations in the topology or disturbances in the system. Systems based on power electronics, such as Flexible Alternate Current Transmission Systems (FACTS) and Line Current Commutated (LCC)- or Voltage Source Converters (VSC)-based High Voltage Direct Current -HVdc- systems have emerged as an important and effective alternative to improve these levels in power systems.

\subsection{VSC-HVdc on Power Systems}

VSC-HVdcs have shown to be effective means to stabilize transmission systems with resulting higher energy transfer capability and reduced risk of line trips. This solution enables power grid owners to increase the existing transmission network capacity while improving the operating margins necessary for grid stability. As a result, a more reliable service can be provided.

Different studies have shown the capability of VSC-HVdc enhancing the stability of power systems [7], [8], providing voltage support [9], [10] and its application in the connection of wind farms [11], [12], just to mention some references.

Currently, about seven VSC-HVdc links operate in the world with a total capacity close to $1300 \mathrm{MW}$. Moreover, four more projects have already been ordered which will mean an additional transmission capacity of $1200 \mathrm{MW}$ by the first semester of 2010 .

\subsection{Why VSC-HVdc}

Transmission System Owners, and in general all different agents, have had to carry out an optimization of their assets in order to achieve a competitive 
position and survive in the new deregulated electricity market [13]. This situation has taken some transmissions networks to levels of high stress conditions affecting increasing the probability of blackouts [14]. On the other hand, customers demand a quality electricity supply from the respective electric agent, including constant voltage, power supply without interruptions and constant frequency.

The objective of the expansion of transmission systems is to increase the capacity of the network and effectively improve the levels of flexibility, reliability and security. Among the possible solutions to achieve this objective, FACTS devices and HVdc systems play an important role. These type of devices/systems have shown to be powerful instruments to stabilize transmission systems with resulting higher energy transfer capability [15], [16].

Most of FACTS devices and thyristor-based HVdc systems only have one degree of freedom; i.e., they are able to control either the active or the reactive power. However Unified Power Flow Controller (UPFC) and VSCHVdc systems are alternatives that have two degrees of freedom. They can control the active and the reactive power, independent of each other. This feature gives UPFC and VSC-HVdc a high potential to remarkably enhance transient stability, provide voltage support, increase damping and control the power flow in a much larger extension compared to other devices/systems.

Even though UPFC and VSC-HVdc have similar characteristics, from the cost point of view VSC-HVdc have the advantage of using conventional power transformers. UPFC requires the installation of power transformer in series with the transmission line, which demands much higher levels of insulation, more complex design, construction and installation details.

VSC-HVdc is becoming a serious candidate when there is a need for expanding the transmission network thanks to the dynamics benefits, the development of turn off capable valves with higher rating values and the possibility to lay the transmission cables underground or underwater [17], [18]. Furthermore the interest for using VSC-HVdc systems to connect offshore wind farms is reaching higher levels every day [19].

The positive impact of VSC-HVdc on the dynamics of power systems, the increasing trend of using these systems in the growing field of wind farms, the effective power flow control when used in synchronous networks, besides the advantages over others FACTS devices and thyristor-based HVdc systems predict that VSC-HVdc will play a very important active role in power systems in the near future. Therefore, it is of high interest to explore, study, analyze and derive different control strategies and possible ways to 
control this type of systems.

\subsection{Applied Methods in the Control Strategies}

In the derivation of the control strategies two different theories are considered: Nonlinear theory and Linear theory. Nonlinear theory is based on Lyapunov theory; more specifically Control Lyapunov Function (CLF). The linear theory is based on small signal analysis. The purpose of using nonlinear theory is to derive a control strategy for enhancing the transient stability of the system. The objective of the linear theory is to formulate a control law for improving the power oscillation damping of the system.

Some authors have used nonlinear theory in the derivation of control strategies for shunt FACTS devices in order to enhance the transient stability as in [20], [21], [22], [23] and [24] to mention some references. Although all of them are referred to FACTS devices either shunt/series, none of them is specifically focused on VSC-HVdc systems.

A large number of authors have used linear theory in the derivation of control strategies for power oscillation damping. Some authors have specifically studied VSC-HVdcs in the increase of power oscillation damping. Some useful references in small signals analysis and references of VSC-HVdcs with application in increase of damping in power systems are [8], [25], [26], [27], [28], and [29]. Some known procedures and methodologies will be followed in the derivation of the control strategy and the selection of the input signal.

\subsection{Multichoice Control Strategy}

The objective of the multichoice control strategy is to have available several input power signals that will enhance the transient stability, damp electromechanical oscillations, provide voltage support or change the power flow condition. When a disturbance occurs in the system, the control can switch to one of several input signals. The output of the control is the controllable variables of the VSC-HVdc. Figure 1.1 shows an scheme of the multichoice control, considering a VSC-HVdc connected between bus $i$ and bus $j$, where:

$\Delta P_{1}$ : Signal based on nonlinear theory 


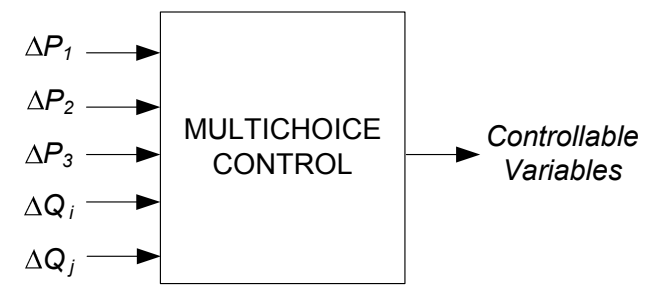

Figure 1.1: Multichoice Control

$\Delta P_{2}$ : Signal based on linear theory

$\Delta P_{3}$ : Signal based on power flow control

$\Delta Q_{i}$ : Signal based on Automatic Voltage Regulator (AVR) and linear theory

$\Delta Q_{j}$ : Signal based on AVR and linear theory

The active power signals are used to enhance the transient stability and increase the damping of the power system. The reactive power signals are mainly used for voltage support at the nodes where the HVdc is connected. Alternatively it is also possible to have a supplementary control of reactive power to improve the damping of the system.

Concerning the active power signals, $\Delta P_{1}$ is primarily intended for enhancing the transient stability of the system. However $\Delta P_{1}$ is also able to provide damping in the system. $\Delta P_{2}$ is primarily intended for improving the damping in the system. However the signal might also enhance the transient stability of the system. The redundancy of the signals increases the reliability of the control.

The reactive power signals are intended to keep the voltage at the buses where the HVdc is connected as close as possible to a reference voltage. Alternatively, the converters can also provide damping by modulating the reactive power. This option might be useful when the dc cable is out of service, but the converters are available.

\subsection{Objectives}

One of the main features of VSC-HVdc systems is the fast and independent control of active and reactive power in each converter station. This charac- 
teristic, which gives VSCs the possibility to operate in all four quadrants, together with an appropriate control strategy allow VSC-HVdcs to enhance transient stability, increase power oscillation damping and provide voltage stability in power systems in a effective way.

The project analyzes the impact of a VSC-HVdc on power system when the VSC-HVdc is connected in a synchronous network. The objectives of the project are:

1. To derive a control strategy for enhancing the transient stability

2. To derive a control strategy for increasing power oscillation damping

3. To derive a control strategy for improving voltage stability

4. To formulate an appropriate operation of a multichoice control input with the control strategies

5. To include a power flow control feature in the multichoice control, since the project considers the connection of the VSC-HVdc in a synchronous system.

\subsection{Outline of the Thesis}

The next five chapters of the thesis are organized as follows:

Chapter 2 describes the derivation of the injection model of a VSC-HVdc. This model is used for deriving and testing the different control strategies and the operation of the multichoice control.

Chapter 3 gives a description of the theory behind the derivation of the control strategies.

Chapter 4 presents the application of the nonlinear theory and linear analysis on VSC-HVdc systems. The chapter initially describes the derivation of the control strategy for enhancing transient stability based on Control Lyapunov Function and next the derivation of the control strategy for improving damping based on modal analysis.

Chapter 5 describes the test power system used in the numerical examples and shows the results of testing the different control strategies and the possible modes of operation of the multichoice control. 
Chapter 6 presents the conclusions of the project and suggests some ideas for future work.

\subsection{Main contributions}

The main contributions of the thesis are:

- Injection Model. Injection Model of VSC-HVdc was derived and evaluated. In several numerical examples the results of the injection model were compared with results of a more detailed model (model of HVdcLight provided by ABB). The dynamic behavior of the power system was very similar.

- Energy function for a VSC-HVdc. Energy function for most systems based on power electronics are available in the literature. However for the specific case of VSC-HVdc there is no prior references in the literature about energy function. In this project an energy function for a power system including VSC-HVdc has been derived.

- Control Lyapunov Function for a VSC-HVdc. A CLF for enhancing the transient stability of the system was derived. As the case of energy functions, CLFs are known for most of the power electronics-based systems. However by the time the CLF for a VSC-HVdc was derived, no prior references in the literature were found.

- Modulation of active and reactive power for enhancing transient stability. It was shown that a higher enhancement of transient stability is achieved when the control strategies that modulate the active power and the control strategy that modulates reactive power (voltage support) are considered simultaneously.

- Enhancement of stability and increase of damping based on CLF- and modal-analysis-based control strategies. It was shown that the sum of the derived control strategy for enhancing transient stability (CLF) and the derived control strategy for increasing power oscillation damping (modal analysis) gives larger transient stability and power oscillation damping than when they are used individually.

- Power flow control. It was shown that a VSC-HVdc connected in a synchronous power system has the ability to rapidly change the power 
flow pattern to secure a post-contingency operating point by selecting the appropriate input signal.

- Multichoice control strategy. A multichoice control strategy for a VSCHVdc has been formulated. The control scheme effectively increases transient stability, power oscillation damping and voltage stability of the power system by combining or by using individually the different inputs (control strategies) of the control. Furthermore this control strategy optimizes the utilization of the transmission system by controlling the power flow, which also results in greater margin of stability in the power system.

\subsection{List of Publications}

The following articles were published during the project:

- H. Latorre, M. Ghandhari and L. Söder, "Control of a VSC-HVdc Operating in Parallel with AC Transmission Lines", IEEE PES Transmission and Distribution, Conference and Exposition, Caracas, Venezuela, Aug. 2006.

- H. Latorre, M. Ghandhari and L. Söder, "Application of Control Lyapunov Functions to Voltage Source Converters-based High Voltage Direct Current for Improving Transient Stability", Power Tech Conf., Lausanne, Switzerland, Jul. 2007.

- H. Latorre, M. Ghandhari and L. Söder, "Multichoice Control Strategy for VSC-HVdc", 2007 iREP Symposium, Charleston, South Carolina, USA, Aug. 2007.

- H. Latorre, M. Ghandhari and L. Söder, "Active and Reactive Power Control of a VSC-HVdc", Electric Power Systems Research, Mar. 2008. 


\section{Chapter 2}

\section{Modeling of VSC-HVdc}

\section{$2.1 \quad$ VSC-HVdc}

VSCs are based on valves that can be switched on and off by a control signal. By choice of the switching instant it is possible to generate any desired wave shape. With higher switching frequency components it is possible to use Pulse Width Modulation (PWM) technology to re-create the ac voltage. With PWM it is possible to create any phase angle or voltage amplitude (within certain limit) by changing the PWM pattern, which can be done almost instantaneous. Hereby PWM offers the possibility to control both active and reactive power independently.

The control of the voltage magnitude and the phase angle of the converters makes the use of separate control for active and reactive power possible. The active power loop can be set to control either the active power or the dc-side voltage. In a dc link, one station will then be selected to control the active power while the other must be set to control the dc-side voltage. The reactive power control loop can be set to control either the reactive power or the ac-side voltage. Either of these two modes can be selected independently at either end of the dc link [30].

Figure 2.1 shows a simple single line diagram of the basic components of a VSC-HVdc system. The main components are the bridge of valves with turn on/off capability (e.g., IGBTs or GTOs); a converter reactor that separates the ac fundamental frequency from the raw PWM waveform; a conventional high power transformer in case the desired dc voltage does not match the ac system voltage; a high frequency harmonic filter (typical value of switching 
frequency of the converter is $2 \mathrm{kHz}$ ) and a circuit breaker. On the dc side the main components are the dc capacitor which serves as dc filter and the cables [17].

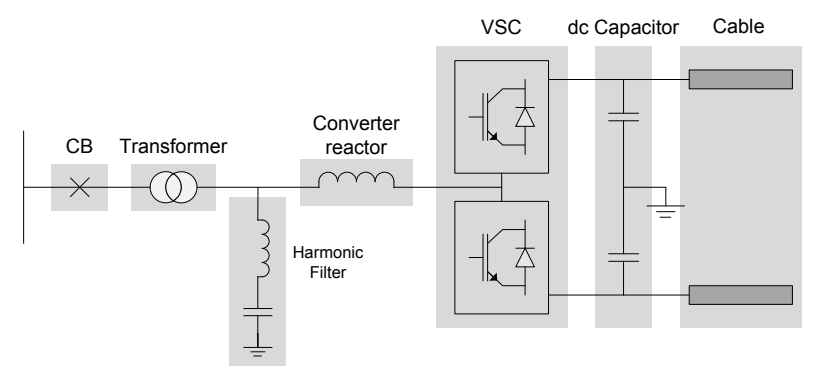

Figure 2.1: Simplified single line diagram for VSC-HVdc

HVdc transmission with VSCs can be beneficial to overall system performance. VSC technology can rapidly control both active and reactive power independently of one another. Reactive power can also be controlled at each terminal independent of the dc transmission voltage level. This control capability gives total flexibility to place converters anywhere in the ac network since there is no restriction on minimum network short-circuit capacity. Self commutation with VSC even permits black starts; i.e., the converter can be used to synthesize a balanced set of three phase voltages like a virtual synchronous generator. The dynamic support of the ac voltage at each converter terminal improves the voltage stability and can increase the transfer capability of the sending- and receiving-end ac systems, thereby leveraging the transfer capability of the dc link. Unlike conventional HVdc transmission, the converters themselves have no reactive power demand and can actually control their reactive power to regulate the ac system voltage just like a generator.

Active and reactive power can be controlled by changing the phase angle of the converter ac voltage with respect to the phase angle at the bus of connection of the HVdc, and the magnitude of the fundamental component of the converter ac voltage. By controlling these two aspects of the converter, operation in all quadrants is possible. This means that the converter can be operated in the middle of its reactive power range near unity power factor to maintain dynamic reactive power reserve for contingency voltage support similar to a STATic synchronous COMpensator (STATCOM). It also means 
that the real power transfer can be exchanged with the ac network or waiting for switching of power compensation. Figure 2.2 shows the restricted operating area of a P-Q diagram due to the limitation on the dc cable and dc voltage [17].

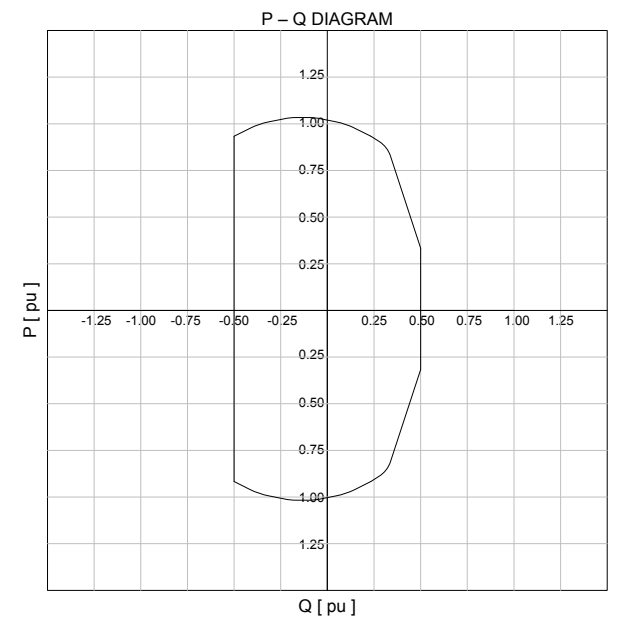

Figure 2.2: $\mathrm{P}$ - Q diagram for the whole dc voltage range

The advantages of VSC-HVdc systems can be summarized as follows [31]:

- Simultaneous control of both active and reactive power. The ac voltage can be controlled at both stations.

- No need for short circuit power for commutation.

- Black start capability.

- Can operate without communication between the stations.

- No change of voltage polarity when the power direction is changed.

- Possibility to use robust and economical extruded cables in both land and sea.

- Small converters that reduce the requirements for space.

- Simple interface with ac system. 
- Compact filters.

- Variable frequency.

- Only conventional ac transformers are required

- Increase transfer of power in parallel ac lines

\subsection{Injection Model of VSC-HVdc}

The injection model is intended for analysis of power flows and electromechanical dynamics in a power system. For this reason it is sufficient to consider the power frequency components of voltages and currents in the ac system. The injection model can be considered as an element in a power system, which provides adequate interaction with other system elements for exploring dynamic performance and stability of the system as a whole. Harmonics and dc transient components are neglected, since they normally have a second order effect on the active and reactive powers. Voltages and currents are represented by phasors in the ac network, which vary with time during transients. The injection model is valid for symmetrical conditions, i.e., for positive sequence voltages and currents. The model is used in the derivation and test of the control strategies.

Figure 2.3 shows a basic structure of a VSC-HVdc link connected in parallel with an ac transmission line.

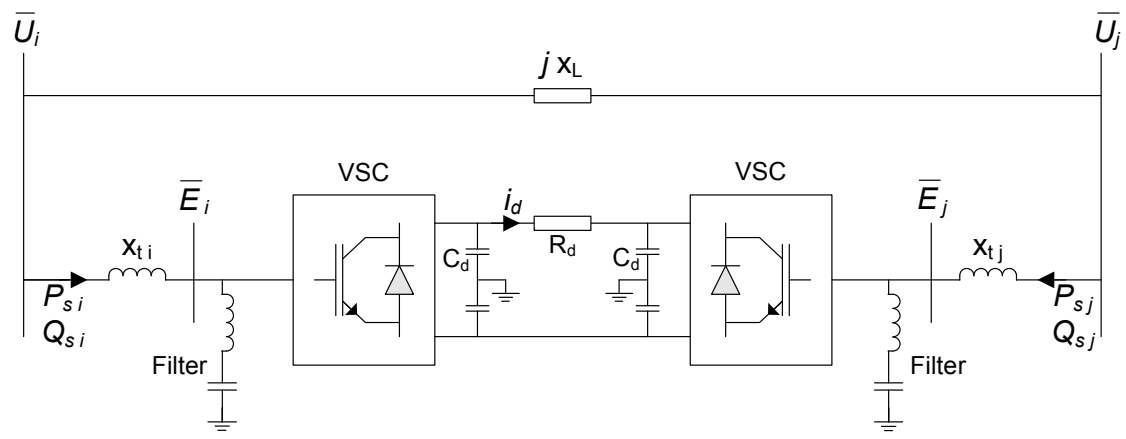

Figure 2.3: Basic Structure of VSC-HVdc.

From the nodes of connections bus $i$ and bus $j$, a VSC-HVdc can be seen as a synchronous machine without inertia where the production or consump- 
tion of the active power is independent of the production or consumption of the reactive power. This interpretation leads to modeling a VSC-HVdc as two controllable voltage sources, as shown in Figure 2.4, in series with a reactance, which represents the impedance of the power transformer.

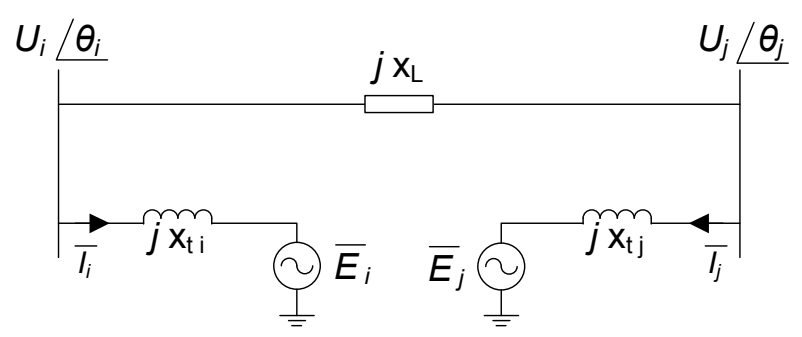

Figure 2.4: Simple model of a VSC-HVdc.

The controllable voltage sources, $\bar{E}_{i}$ and $\bar{E}_{j}$ are defined as

$$
\bar{E}_{i}=E_{i} e^{j \gamma_{i}} \quad \bar{E}_{j}=E_{j} e^{j \gamma_{j}}
$$

where $E_{i}, E_{j}, \gamma_{i}$ and $\gamma_{j}$ are the controllable variables (magnitude and phase angle, respectively) of the voltage sources. $x_{t i}$ and $x_{t j}$ are the reactances of the power transformers.

If harmonics are ignored the injection of power into the HVdc is defined by:

$$
\begin{array}{rlrl}
P_{s i} & =\frac{U_{i} E_{i} \sin \left(\theta_{i}-\gamma_{i}\right)}{x_{t i}} & Q_{s i} & =\frac{U_{i}\left[U_{i}-U_{i} E_{i} \cos \left(\theta_{i}-\gamma_{i}\right)\right]}{x_{t i}} \\
P_{s j}=\frac{U_{j} E_{j} \sin \left(\theta_{j}-\gamma_{j}\right)}{x_{t j}} & Q_{s j}=\frac{U_{j}\left[U_{j}-U_{j} E_{j} \cos \left(\theta_{j}-\gamma_{j}\right)\right]}{x_{t j}}
\end{array}
$$

From equation (2.2) can be concluded that $P_{s i}, Q_{s i}$ can be independently controlled by $\gamma_{i}$ and $E_{i}$. Likewise $P_{s j}, Q_{s j}$ can be controlled by $\gamma_{j}$ and $E_{j}$.

It is assumed that the dc voltage control keeps the dc voltage magnitude close to the rated voltage. Therefore the losses of the converters are assumed constant, regardless of the current through the converters. The losses are consequently represented as a constant active load. The losses of the dc cables are neglected. The relation between bus $i$ and bus $j$ is given by the active power: $P_{s i}=-P_{s j}$; the reactive power is independent at each bus. Figure 2.5 shows the injection model. 


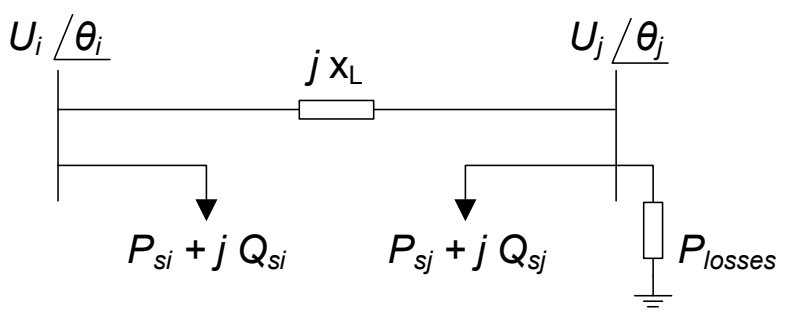

Figure 2.5: Injection model of a VSC-HVdc.

The injected power into the VSC-HVdc can be re-written as:

$$
\bar{S}_{s i}=\bar{U}_{i}\left(\bar{I}_{i}\right)^{*}=P_{s i}+j Q_{s i}
$$

where

$$
\begin{aligned}
P_{s i} & =b_{t i} U_{i} E_{i} \sin \left(\theta_{i}-\gamma_{i}\right) \\
& =b_{t i} U_{i} E_{i}\left(\sin \theta_{i} \cos \gamma_{i}-\cos \theta_{i} \sin \gamma_{i}\right) \\
Q_{s i} & =b_{t i}\left[U_{i}^{2}-U_{i} E_{i} \cos \left(\theta_{i}-\gamma_{i}\right)\right] \\
& =b_{t i}\left[U_{i}^{2}-U_{i} E_{i}\left(\cos \theta_{i} \cos \gamma_{i}+\sin \theta_{i} \sin \gamma_{i}\right)\right]
\end{aligned}
$$

and,

$$
b_{t i}=1 / x_{t i}
$$

Similar procedure followed at node $j$ gives:

$$
\begin{aligned}
P_{s j} & =b_{t j} U_{j} E_{j}\left(\sin \theta_{j} \cos \gamma_{j}-\cos \theta_{j} \sin \gamma_{j}\right) \\
Q_{s j} & =b_{t j}\left[U_{j}^{2}-U_{j} E_{j}\left(\cos \theta_{j} \cos \gamma_{j}+\sin \theta_{j} \sin \gamma_{j}\right)\right]
\end{aligned}
$$

By introducing the following variables:

$$
\begin{array}{ll}
E_{i}^{r e}=E_{i} \cos \gamma_{i} & E_{i}^{i m}=E_{i} \sin \gamma_{i} \\
E_{j}^{r e}=E_{j} \cos \gamma_{j} & E_{j}^{i m}=E_{j} \sin \gamma_{j}
\end{array}
$$


the injected power becomes:

$$
\begin{aligned}
\bar{S}_{s i} & =P_{s i}+j Q_{s i} \\
P_{s i} & =b_{t i} U_{i}\left(\sin \theta_{i} E_{i}^{r e}-\cos \theta_{i} E_{i}^{i m}\right) \\
Q_{s i} & =b_{t i}\left[U_{i}^{2}-U_{i}\left(\cos \theta_{i} E_{i}^{r e}+\sin \theta_{i} E_{i}^{i m}\right)\right] \\
\bar{S}_{s j} & =P_{s j}+j Q_{s j} \\
P_{s j} & =b_{t j} U_{j}\left(\sin \theta_{j} E_{j}^{r e}-\cos \theta_{j} E_{j}^{i m}\right) \\
Q_{s j} & =b_{t j}\left[U_{j}^{2}-U_{j}\left(\cos \theta_{j} E_{j}^{r e}+\sin \theta_{j} E_{j}^{i m}\right)\right] \\
P_{s i} & =-P_{s j}
\end{aligned}
$$

From equation (2.4) it is possible to observe:

$$
\begin{array}{ll}
E_{i}=\sqrt{\left(E_{i}^{r e}\right)^{2}+\left(E_{i}^{i m}\right)^{2}} & \gamma_{i}=\arctan \left(\frac{E_{i}^{i m}}{E_{i}^{r e}}\right) \\
E_{j}=\sqrt{\left(E_{j}^{r e}\right)^{2}+\left(E_{j}^{i m}\right)^{2}} & \gamma_{j}=\arctan \left(\frac{E_{j}^{i m}}{E_{j}^{r e}}\right)
\end{array}
$$

Equation (2.4) can be divided into two terms, expressing the fixed variables (or set values) $E_{i o}^{r e}, E_{i o}^{i m}, E_{j o}^{r e}$ and $E_{j o}^{i m}$; and the modulated variables $\Delta E_{i}^{r e}$, $\Delta E_{i}^{i m}, \Delta E_{j}^{r e}$ and $\Delta E_{j}^{i m}$.

$$
\begin{array}{ll}
E_{i}^{r e}=E_{i o}^{r e}+\Delta E_{i}^{r e} & E_{i}^{i m}=E_{i o}^{i m}+\Delta E_{i}^{i m} \\
E_{j}^{r e}=E_{j o}^{r e}+\Delta E_{j}^{r e} & E_{j}^{i m}=E_{j o}^{i m}+\Delta E_{j}^{i m}
\end{array}
$$

Thus, expressions of active and reactive power in equation (2.5), can be written as:

$$
\begin{aligned}
P_{s i} & =b_{t i} U_{i}\left[\sin \theta_{i}\left(E_{i o}^{r e}+\Delta E_{i}^{r e}\right)-\cos \theta_{i}\left(E_{i o}^{i m}+\Delta E_{i}^{i m}\right)\right] \\
& =b_{t i} U_{i}\left(\sin \theta_{i} E_{i o}^{r e}-\cos \theta_{i} E_{i o}^{i m}\right)+b_{t i} U_{i}\left(\sin \theta_{i} \Delta E_{i}^{r e}-\cos \theta_{i} \Delta E_{i}^{i m}\right) \\
& =P_{s i o}+\Delta P_{s i}
\end{aligned}
$$


and similarly for $Q_{s i}, P_{s j}$ and $Q_{s j}$

$$
\begin{aligned}
P_{s i} & =P_{s i o}+\Delta P_{s i} \\
Q_{s i} & =Q_{s i o}+\Delta Q_{s i} \\
P_{s j} & =P_{s j o}+\Delta P_{s j} \\
Q_{s j} & =Q_{s j o}+\Delta Q_{s j}
\end{aligned}
$$

where the subindex $o$ denotes the uncontrolled $P$ and $Q . \Delta P$ and $\Delta Q$ correspond to the modulative parts.

By Considering equations (2.5) and (2.7), the right hand side terms in equation (2.8) become:

$$
\begin{aligned}
P_{s i o} & =b_{t i} U_{i}\left(\sin \theta_{i} E_{i o}^{r e}-\cos \theta_{i} E_{i o}^{i m}\right) \\
Q_{s i o} & =b_{t i}\left[U_{i}^{2}-U_{i}\left(\cos \theta_{i} E_{i o}^{r e}+\sin \theta_{i} E_{i o}^{i m}\right)\right] \\
P_{s j o} & =b_{t j} U_{j}\left(\sin \theta_{j} E_{j o}^{r e}-\cos \theta_{j} E_{j o}^{i m}\right) \\
Q_{s j o} & =b_{t j}\left[U_{j}^{2}-U_{j}\left(\cos \theta_{j} E_{j o}^{r e}+\sin \theta_{j} E_{j o}^{i m}\right)\right] \\
\Delta P_{s i} & =b_{t i} U_{i}\left(\sin \theta_{i} \Delta E_{i}^{r e}-\cos \theta_{i} \Delta E_{i}^{i m}\right) \\
\Delta Q_{s i} & =-b_{t i} U_{i}\left(\cos \theta_{i} \Delta E_{i}^{r e}+\sin \theta_{i} \Delta E_{i}^{i m}\right) \\
\Delta P_{s j} & =b_{t j} U_{j}\left(\sin \theta_{j} \Delta E_{j}^{r e}-\cos \theta_{j} \Delta E_{j}^{i m}\right) \\
\Delta Q_{s j} & =-b_{t j} U_{j}\left(\cos \theta_{j} \Delta E_{j}^{r e}+\sin \theta_{j} \Delta E_{j}^{i m}\right)
\end{aligned}
$$

Note however that

$$
\begin{aligned}
P_{s j o} & =-P_{s i o} \\
\Delta P_{s j} & =-\Delta P_{s i}
\end{aligned}
$$




\section{Chapter 3}

\section{Theoretical Background}

\subsection{Power System Modeling}

The basic structure of a electrical power system is divided in three main parts: Generation, Transmission and Distribution. The different parts of the power system operate at different voltage levels. Typically generation and distribution are classified within the medium voltage range (between $1 \mathrm{kV}$ and $100 \mathrm{kV}$ ). Transmission is classified in high voltage (between $100 \mathrm{kV}$ and $300 \mathrm{kV}$ ) or extra-high voltage (between $300 \mathrm{kV}$ and above).

Generation is produced by converting the mechanical energy appearing on the output shaft of a turbine into electrical energy. This conversion is almost universally done by the use of synchronous generator. The synchronous generator feeds its electrical power into the transmission system via a step-up transformer.

A significant advantage of electrical energy is that it can be generated near to the primary energy resource and then be transmitted over long distances to the load centers. The energy lost in a transmission system is proportional to the square of the current. For this reason the transmission lines operate at high or extra-high voltage. The transmission network connects the power stations into one system and transmits and distributes power to load centers in an optimal way.

The demand for electrical power is never constant and changes continuously throughout the day and time. The changes in demand of individual consumers may be fast and frequent. However, the higher the level of voltage from where the consumers are seen, the smaller and smoother are the 
changes. Consequently, the total power demand at the transmission level changes in a more or less predictable way.

\subsubsection{Synchronous Machines}

A synchronous generator consists basically of a stator (or armature), on which the three-phase armature winding is normally wound, and a rotor (or field), on which the dc field winding is wound. The rotor can also be equipped with additional short-circuited damper windings to reduce the mechanical oscillations of the rotor. The stator is represented by three magnetic axes, $a$, $b$ and $c$ each corresponding to one of the phase windings. On the other hand, the rotor is represented by two axis, namely the direct axis ( $d$-axis), which is the magnetic axis of the field winding, and the quadrature axis ( $q$-axis), which is the axis of symmetry between two poles.

In the analysis of dynamics of power systems, synchronous machines are typically represented by three models, namely: Two-Axis model, Flux-Decay model (or One-Axis model) and Classical model.

For transient stability analysis, synchronous machines are normally simplified by making the following assumptions [32]:

1. The stator transients are neglected.

2. All the stator resistances are neglected.

3. During the transient-state, the rotor speed is near synchronous speed.

4. The rotor transient saliency is neglected.

In the Two-Axis model it is assumed that the $q$-axis is equipped with a short-circuited damper winding. Under the mentioned assumptions, the dynamics of generators using this model are represented by the following four state equations: 


$$
\begin{aligned}
\dot{\delta} & =\omega \\
\dot{\omega} & =\frac{1}{M}\left(P_{m}-P_{e}-D \omega\right) \\
\dot{E}_{q}^{\prime} & =\frac{1}{T_{d o}^{\prime}}\left(E_{f}-\frac{x_{d}}{x_{d}^{\prime}} E_{q}^{\prime}+\frac{x_{d}-x_{d}^{\prime}}{x_{d}^{\prime}} U \cos (\delta-\theta)\right) \\
\dot{E}_{d}^{\prime} & =\frac{1}{T_{q o}^{\prime}}\left(-\frac{x_{q}}{x_{q}^{\prime}} E_{d}^{\prime}-\frac{x_{q}-x_{q}^{\prime}}{x_{q}^{\prime}} U \sin (\delta-\theta)\right)
\end{aligned}
$$

and $P_{e}$ given by

$$
P_{e}=\frac{E_{q}^{\prime} U \sin (\delta-\theta)+E_{d}^{\prime} U \cos (\delta-\theta)}{x_{d}^{\prime}}
$$

where,

$\delta$ is the angular position of the rotor relative to a reference synchronous machine with synchronous speed

$\omega$ is the rotor speed

$E_{q}^{\prime}$ is the $q$-axis transient emf

$E_{d}^{\prime}$ is the $d$-axis transient emf

$P_{m}$ is the mechanical power

$P_{e}$ is the electrical power

$D$ is the damping constant

$M$ is the inertia constant

$E_{f}$ is an emf proportional to the field voltage

$x_{d}$ is the $d$-axis synchronous reactance

$x_{d}^{\prime}$ is the $d$-axis transient reactance

$x_{q}$ is the $q$-axis synchronous reactance 
$x_{q}^{\prime}$ is the $q$-axis transient reactance

$T_{d o}^{\prime}$ is the $d$-axis transient open-circuit time constant

$T_{q o}^{\prime}$ is the $q$-axis transient open-circuit time constant

$U$ is the magnitude of the voltage of the network where the synchronous generator is connected

$\theta$ is the phase angle of $U$

The One-Axis model does not consider the dynamics due to the damperwinding. In this way, the dynamics of the Flux-Decay model are described by equation (3.1) - equation (3.3), where $P_{e}$ is given by:

$$
P_{e}=\frac{E_{q}^{\prime} U \sin (\delta-\theta)}{x_{d}^{\prime}}
$$

The classical model is the simplest of all the synchronous machine models. It is also called the constant voltage behind the transient reactance $x_{d}^{\prime}$ model. In this model the flux produced by the field winding and the current through field winding are assumed to be constant. This assumption results in a second-order system described by equation (3.1) and equation (3.2), where $P_{e}$ is given by equation (3.6).

\subsubsection{Static Load}

A typical power system may consists of several hundreds buses at the transmission level, but there could be a hundred thousand buses at the distribution levels. For this reason, when power systems are analyzed only the transmission level is considered. The distribution networks are replaced by equivalent loads, referred to as composite loads.

The demand of the composite load normally depends on the bus voltage and the frequency. The functions describing the dependency of the active and reactive load demand on the voltage and frequency $P(U, f)$ and $Q(U, f)$ are called the static load characteristics. The characteristics $P(U)$ and $Q(U)$ taken at constant frequency are called the voltage characteristics while the characteristics $P(f)$ and $Q(f)$ taken at constant voltage are called the frequency characteristics. The characteristics of the composite loads depend on the characteristics of their individual components. 
The determination of a simple and valid composite load models is not an easy task and it is still subject of intensive research. Composite loads may be represented by static or dynamic models or combination the modes. The following models are the most popular modes used in analysis of power systems [33].

\section{Constant Power, Current or Impedance}

The simplest load models assume one of the following features:

- a constant power demand $(P)$

- a constant current demand $(I)$

- a constant impedance $(Z)$

A constant power model is voltage invariant and allows loads with a stiff voltage characteristics. This model is often used in load flow calculations, but it is generally unsatisfactory for transient stability analysis, in the presence of large voltage variations. The constant current model gives a load demand that changes linearly with voltage and is a reasonable representation of the real power demand of a mix of resistive and motor devices. The constant impedance model the load power changes proportionally to the squared of the voltage and represents some lighting loads well, but it does not model stiff loads at all well. To obtain a more general voltage characteristics, the benefits of each of these characteristics can be combined by using the so-called polynomial or ZIP model consisting of the sum of the constant impedance $(Z)$, constant current $(I)$, and constant power $(P)$ terms:

$$
\begin{aligned}
& P=P_{o}\left[a_{1}\left(\frac{U}{U_{o}}\right)^{2}+a_{2}\left(\frac{U}{U_{o}}\right)+a_{3}\right] \\
& Q=Q_{o}\left[a_{4}\left(\frac{U}{U_{o}}\right)^{2}+a_{4}\left(\frac{U}{U_{o}}\right)+a_{6}\right]
\end{aligned}
$$

where, $U_{o}, P_{o}$ and $Q_{o}$ are normally taken as the values at initial operating conditions. The parameters of this polynomial model are the coefficients $\left(a_{1}\right.$ to $a_{6}$ ) and the power factor of the load. 


\section{Exponential Load Model}

In this model the power is related to the voltage by:

$$
\begin{aligned}
& P=P_{o}\left(\frac{U}{U_{o}}\right)^{m p} \\
& Q=Q_{o}\left(\frac{U}{U_{o}}\right)^{m q}
\end{aligned}
$$

where, $m p$ and $m q$ are the parameters of the model. Note that by setting the parameters to 0,1 or 2 the load can be represented by constant power, constant current or constant impedance, respectively.

\section{Frequency-Dependent Load Model}

Frequency dependence is usually represented by multiplying either a polynomial or exponential load model by a factor $\left[1+a_{f}\left(f-f_{o}\right)\right]$, where $f$ is the actual frequency, $f_{o}$ is the rated frequency and $a_{f}$ is the model frequency sensitivity parameter.

\subsubsection{Multi-Machine Power System}

Power systems consist of several transmission lines which interconnect the generator stations with the load centers. Since electrical energy cannot be stored in large quantities, there is always a balance between the total power generated and the total power consumed.

Let us consider a multi-machine power system with $n$ generators and a transmission network with $N$ buses. Furthermore, consider the following assumptions:

1. Generators are described by one-axis model

2. Mechanical power of each generator, $P_{m}$, is constant

3. Loads are represented by static model

4. Transmission networks is lossless.

Based on the first assumption the generator can be modeled as shown in Figure 3.1, where the reactance of the $k$ :th step-up transformer is included 
in $x_{d k}^{\prime}$. The voltage at the internal bus is given by the magnitude $E_{q k}^{\prime}$ and the phase angle $\delta_{k}$

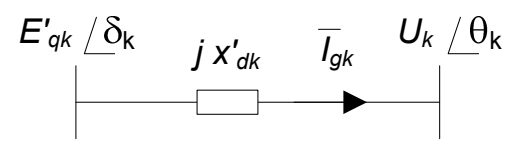

Figure 3.1: Synchronous generator one-axis dynamic circuit

Let $\bar{Y}_{\text {bus }}$ of order $(N \times N)$ be the admittance matrix of the transmission network. Since the transmission network is lossless, the $k l$ :th element of the admittance matrix is defined by $\bar{Y}_{b u s_{k l}}=j B_{k l}$.

The complex power supplied by the generator $k$ injected into its terminal transmission network bus $k$ is defined by:

$$
\begin{aligned}
P_{g k} & =\operatorname{Re}\left(\bar{U}_{k} \bar{I}_{g k}^{*}\right) \\
& =\frac{E_{q k}^{\prime} U_{k} \sin \left(\delta_{k}-\theta_{k}\right)}{x_{d k}^{\prime}} \\
Q_{g k} & =\operatorname{Im}\left(\bar{U}_{k} \bar{I}_{g k}^{*}\right) \\
& =\frac{E_{q k}^{\prime} U_{k} \cos \left(\theta_{k}-\delta_{k}\right)-U_{k}^{2}}{x_{d k}^{\prime}}
\end{aligned}
$$

Concerning the transmission network, the complex power injected into bus $k$ is defined by:

for $k=1, \ldots, n$

$$
\begin{aligned}
P_{k} & =\sum_{l=1}^{N} B_{k l} U_{k} U_{l} \sin \left(\theta_{k}-\theta_{l}\right)+\frac{E_{q k}^{\prime} U_{k} \sin \left(\theta_{k}-\delta_{k}\right)}{x_{d k}^{\prime}} \\
Q_{k} & =-\sum_{l=1}^{N} B_{k l} U_{k} U_{l} \cos \left(\theta_{k}-\theta_{l}\right)+\frac{U_{k}^{2}-E_{q k}^{\prime} U_{k} \cos \left(\theta_{k}-\delta_{k}\right)}{x_{d k}^{\prime}}
\end{aligned}
$$

and for $k=(n+1), \ldots, N$ 


$$
\begin{aligned}
P_{k} & =\sum_{l=1}^{N} B_{k l} U_{k} U_{l} \sin \left(\theta_{k}-\theta_{l}\right) \\
Q_{k} & =-\sum_{l=1}^{N} B_{k l} U_{k} U_{l} \cos \left(\theta_{k}-\theta_{l}\right)
\end{aligned}
$$

Let $P_{L k}+j Q_{L k}$ be the complex power of the load at bus $k$. Then, for $k=1, \ldots, N$, the power flow equations (3.10) and (3.11) can be written as:

$$
\begin{aligned}
& 0=P_{k}+P_{L k} \\
& 0=Q_{k}+Q_{L k}
\end{aligned}
$$

Since generators are assumed to be one-axis modeled, let the vectors $x$ and $y$ be:

$$
\begin{aligned}
x & =\left[\delta_{1} \ldots \delta_{n}, \omega_{1} \ldots \omega_{n}, E_{q 1}^{\prime} \ldots E_{q n}^{\prime}\right]^{\mathrm{T}} \\
y & =\left[\theta_{1} \ldots \theta_{N}, U_{1} \ldots U_{N}\right]^{\mathrm{T}}
\end{aligned}
$$

then equation (3.1) - equation (3.3) and equation (3.12) can be rewritten as:

$$
\begin{aligned}
\dot{x} & =f(x, y) \\
\dot{0} & =g(x, y)
\end{aligned}
$$

The multi-machine power system is therefore described by a set of differential equations related to the generators and a set of algebraic equations related to the transmission network (balance of power). This dynamic model given by the set of differential-algebraic equations is known as Structure Preserving Model (SPM).

\subsection{Lyapunov Theory}

It is common to model dynamical systems with finite number of coupled first-order ordinary differential equations: 


$$
\begin{aligned}
\dot{x}_{1}= & f_{1}\left(t, x_{1}, \ldots x_{n_{x}}, u_{1} \ldots u_{r}\right) \\
\vdots & \vdots \\
\dot{x}_{n_{x}} & =f_{n}\left(t, x_{1}, \ldots x_{n_{x}}, u_{1} \ldots u_{r}\right)
\end{aligned}
$$

where $\dot{x}_{i}$ denotes the derivative of $x_{i}$ with respect to the time variable $t$ and $u_{1} \ldots u_{r}$ are specified input variables. By using vector notation, equation (3.14) can be written in the following compact form:

$$
x=\left[\begin{array}{c}
x_{1} \\
\vdots \\
x_{n_{x}}
\end{array}\right], \quad u=\left[\begin{array}{c}
u_{1} \\
\vdots \\
u_{p}
\end{array}\right], \quad f(t, x, u)=\left[\begin{array}{c}
f_{1}(t, x, u) \\
\vdots \\
f_{n_{x}}(t, x, u)
\end{array}\right]
$$

Thus, the $n_{x}$ first-order differential equation can be rewritten as one $n_{x^{-}}$ dimensional first-order vector differential equation:

$$
\dot{x}=f(t, x, u)
$$

Equation (3.15) is called the state equation and refer to $x$ as the state and $u$ as the input.

When the state equation does not have an explicit presence of an input $u$, the equation is called the unforced state equation

$$
\dot{x}=f(t, x)
$$

Working with an unforced state equation does not necessarily mean that the input to the system is zero. It could be that the input has been specified as a given function of time, $u=\gamma(t)$, a given feedback function of the state, $u=\gamma(x)$, or both, $u=\gamma(t, x)$. Replacing $u=\gamma$ in (3.15) eliminates $u$ and yields an unforced state equation.

A special case of (3.16) arises when the function $f$ does not depend explicitly on $t$, that is,

$$
\dot{x}=f(x)
$$

in which case the system is said to be autonomous or time invariant. The behavior of an autonomous systems is invariant to shifts in time origin, since changing the time variable from $t$ to $\tau=t-a$ does not change the right-hand 
side of the state equation. If the system is not autonomous, the it is called nonautonomous or time varying.

A point $x=x_{o}$ in the state space is said to be an equilibrium point of equation (3.15) if it has the property that whenever the state of the system starts at $x_{o}$, it will remain at $x_{o}$ for all future time. For the autonomous system (3.17), an equilibrium point $x_{o}$ is defined by the equation

$$
f\left(x_{o}\right)=0
$$

The point $x_{o}$ is Lyapunov stable (or stable in the sense of Lyapunov) if solutions that start near $x_{o}$ remain near $x_{o}$ for all $t \geq 0$. More precisely, an equilibrium point $x_{o}$ is Lyapunov stable if for any $\epsilon>0$ there exists a $\delta=\delta(\epsilon)>0$ such that for every $x(0)$ in which

$$
\left\|x(0)-x_{o}\right\|<\delta
$$

the solution $x(t)$ satisfies

$$
\left\|x(t)-x_{o}\right\|<\epsilon \quad, \quad \forall t>0
$$

if in addition

$$
\left\|x(t)-x_{o}\right\| \rightarrow 0 \quad \text { as } \quad t \rightarrow \infty
$$

then $x_{o}$ is asymptotically stable. An equilibrium point $x_{o}$ that is not stable is called unstable [34].

The method is closely related to the energy of a system. If the rate of change $d E(x) / d t$ of the energy $E(x)$ of an isolated physical system is negative for every possible state $x$, except for a single equilibrium state, $x_{o}$, the energy will then continuously decrease until it finally assumes its minimum value $E\left(x_{o}\right)$. In other words, a dissipative system perturbed from its equilibrium point will always return to it. Thus, by examining the time derivative of $E$ along the trajectories of the system, it is possible to determine the stability of the equilibrium point.

Let us consider again the system (3.17) and let the equilibrium point $x_{o}$ be the origin, that is, $x_{o}=0$. The stability of any other equilibrium point can be obtained by simply translating the coordinate system so that the equilibrium of interest is at the origin in the new coordinates. Let $\mathcal{V}: \mathcal{D} \rightarrow R$ be a continuously differentiable function defined in a domain $\mathcal{D} \subset R^{n_{x}}$ that 
contains the origin. The time derivative of $\mathcal{V}$ along the trajectories of (3.17) (denoted by $\dot{\mathcal{V}}$ ) is given by

$$
\begin{aligned}
& \dot{\mathcal{V}}=\sum_{i=1}^{n_{x}} \frac{\partial \mathcal{V}}{\partial x_{i}} f_{i}(x) \\
& \dot{\mathcal{V}}=\left[\frac{\partial \mathcal{V}}{\partial x_{1}}, \frac{\partial \mathcal{V}}{\partial x_{2}}, \ldots, \frac{\partial \mathcal{V}}{\partial x_{n_{x}}}\right] \cdot\left[\begin{array}{c}
f_{1}(x) \\
f_{2}(x) \\
\vdots \\
f_{n_{x}}(x)
\end{array}\right] \\
& \dot{\mathcal{V}}=\frac{\partial \mathcal{V}}{\partial x} \cdot f(x) \\
& \dot{\mathcal{V}}=\nabla \mathcal{V}(x) \cdot f(x)
\end{aligned}
$$

The time derivative of $\mathcal{V}$ along the trajectories of a system is dependent on the system's equation. Hence, $\dot{\mathcal{V}}$ will be different for different systems.

Theorem 1 Let $x_{o}$ be an equilibrium point for system (3.17) and $\mathcal{D} \subset R^{n_{x}}$ be a domain containing $x_{o}$. Let $\mathcal{V}: \mathcal{D} \rightarrow R$ be a continuously differentiable function such that

$$
\begin{aligned}
& \mathcal{V}\left(x_{o}\right)=0 \\
& \mathcal{V}(x)>0 \quad \forall x \in \mathcal{D}, \text { except at } x_{o} \\
& \dot{\mathcal{V}}(x)=\nabla \mathcal{V}(x) \cdot f(x) \leq 0 \quad \forall x \in \mathcal{D}
\end{aligned}
$$

then the origin is stable. Moreover, if

$$
\dot{\mathcal{V}}(x)=\nabla \mathcal{V}(x) \cdot f(x)<0 \quad \forall x \in \mathcal{D} \text { except at } x_{o} \text { where } \dot{\mathcal{V}}\left(x_{o}\right)=0
$$

or, alternatively

$$
\begin{array}{r}
\dot{\mathcal{V}}(x)=\nabla \mathcal{V}(x) \cdot f(x) \leq 0, \text { provided that } \dot{\mathcal{V}}(x) \text { is not identically } \\
\text { zero on any solution } x(t) \text { in } \mathcal{D} \text {, except at } x_{o} .
\end{array}
$$

then the origin is asymptotically stable 
This theorem is called Lyapunov's stability theorem. The proof can be found in [34].

A continuously differentiable equation $\mathcal{V}(x)$ satisfying (3.18), and (3.20) (or (3.21)) is called a Lyapunov function.

A function $\mathcal{V}(x)$ satisfying conditions (3.18) and (3.19), that is, $\mathcal{V}\left(x_{o}\right)=0$ and $\mathcal{V}(x)>0$ for $x \neq x_{o}$, is said to be positive definite. If it satisfies the weaker condition $\mathcal{V}(x) \geq 0$ for $x \neq x_{o}$, it is said to be positive semidefinite.

Lyapunov functions for a given system are not unique. Furthermore, while the conditions in Lyapunov's stability theorem are sufficient conditions, they are not constructive conditions; they do not tell how to find a Lyapunov function for a particular system. There are however theorems which establish (at least conceptually) that the given conditions in Theorem 1 are also necessary for stability. Such theorems do not give direct help in the practical search for a Lyapunov function. However, the theorem are useful in using Lyapunov theory to draw conceptual conclusions about the behavior of dynamical system. They have also been a basis for the introduction of Control Lyapunov Function for systems with control input.

Stability of the equilibrium point of the nonlinear system (3.17) can also be defined by examining the linearized system. This approach is known as Lyapunov's first (or indirect) method. Linearizing the nonlinear system (3.17) around an equilibrium point $x_{o}$ results in

$$
\Delta \dot{x}=A \Delta x
$$

where,

$$
A=\left[\frac{\partial f(x)}{\partial x}\right]_{x=x_{o}}=\left[\begin{array}{ccc}
\frac{\partial f_{1}(x)}{\partial x_{1}} & \ldots & \frac{\partial f_{1}(x)}{\partial x_{n_{x}}} \\
\vdots & \ddots & \vdots \\
\frac{\partial f_{n_{x}}(x)}{\partial x_{1}} & \ldots & \frac{\partial f_{n}(x)}{\partial x_{n_{x}}}
\end{array}\right]_{x=x_{o}}
$$

which is also called the Jacobian matrix at $x_{o}$. The eigenvalues of $A$ (i.e., $\lambda_{i}$ ) are obtained by solving

$$
\operatorname{det}(A-\lambda \mathbf{I})=0
$$

where "det" is the determinant and $\mathbf{I}$ is the unity matrix

Theorem 2 Let $x_{o}$ be an equilibrium point for the nonlinear system (3.17).

Then, 
1. $x_{o}$ is asymptotically stable if all eigenvalues of the matrix $A$ have real parts.

2. $x_{0}$ is unstable if any of the eigenvalues of the matrix $A$ has a positive real part

The proof can be found in [34].

Note that Theorem 2 is generally not applicable if some of the eigenvalues have zero real part and the others have negative real parts. Thus, it is possible to draw conclusions about the local stability or instability of an equilibrium point for a nonlinear system (3.17), based on the stability or instability of the linearized system (3.23), provided that none of the eigenvalues of the linearized systems have zero real parts.

\section{Example}

Consider the Single Machine Infinite Bus (SMIB) shown in Figure 3.2 and let the synchronous machine be represented by the classical model.

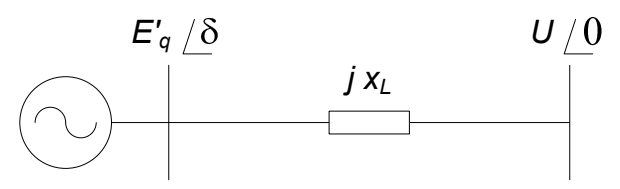

Figure 3.2: SMIB system

The dynamics of the system are given by:

$$
\begin{aligned}
\dot{\delta} & =\omega \\
\dot{\omega} & =\frac{1}{M}\left[P_{m}-P_{\text {emax }} \sin (\delta)-D \omega\right]
\end{aligned}
$$

with

$$
P_{\text {emax }}=\frac{E_{q}^{\prime} U}{x_{d}^{\prime}+x_{t}+x_{L}}
$$

The equilibrium points of the system are given by: 


$$
\begin{aligned}
& \left(\delta_{o 1}, \omega_{o 1}\right)=\left(\arcsin \left(\frac{P_{m}}{P_{\text {emax }}}\right), 0\right) \\
& \left(\delta_{o 2}, \omega_{o 2}\right)=\left(\pi-\arcsin \left(\frac{P_{m}}{P_{\text {emax }}}\right), 0\right) \\
& \left(\delta_{o 3}, \omega_{o 3}\right)=\left(-\pi-\arcsin \left(\frac{P_{m}}{P_{\text {emax }}}\right), 0\right)
\end{aligned}
$$

By applying Theorem 2, it can be found that $\left(\delta_{o 2}, \omega_{o 2}\right)$ and $\left(\delta_{o 3}, \omega_{o 3}\right)$ are unstable. $\left(\delta_{o 1}, \omega_{o 1}\right)$ is an asymptotically stable point. The stability of $x_{o 1}=$ $\left(\delta_{o 1}, \omega_{o 1}\right)$ is examined by applying Theorem 1. For the system (3.24) the following Lyapunov function exists.

$$
\mathcal{V}=\frac{1}{2} M \omega^{2}-P_{m} \delta-P_{\text {emax }} \cos (\delta)+C_{o}
$$

where $C_{o}$ is a constant, such that $\mathcal{V}=0$ at $x_{o 1}$. The time derivative of (3.25) along the trajectories of (3.24) is given by

$$
\begin{aligned}
& \dot{\mathcal{V}}=\nabla \mathcal{V} \cdot f(x) \\
& \dot{\mathcal{V}}=\left[-P_{m}+P_{\text {emax }} \sin \delta \quad M \omega\right] \cdot\left[\begin{array}{c}
\omega \\
\frac{P_{m}-P_{\text {emax }} \sin \delta-D \omega}{M}
\end{array}\right] \\
& \dot{\mathcal{V}}=-D \omega^{2} \leq 0
\end{aligned}
$$

Since $\dot{x}=f(x) \neq 0$, or more precisely,

$$
\dot{\omega}=\frac{1}{M}\left[P_{m}-P_{\text {emax }} \sin (\delta)-D \omega\right] \neq 0
$$

on $\omega=0$ except at $x_{o 1}$, conditions (3.18) - (3.22) are fulfilled. Thus, the equilibrium point $x_{o 1}=\left(\delta_{o 1}, \omega_{o 1}\right)$ is asymptotically stable.

\subsection{Application of Lyapunov Theory to Multi-Machine Power Systems}

In section 3.1.3 the power system was represented by the SPM, which is mathematically described by a set of Differential-Algebraic Equations 


\subsection{APPLICATION OF LYAPUNOV THEORY TO MULTI-MACHINE}

POWER SYSTEMS

(DAE), equation (3.13). The algebraic states $y$ are related to the dynamic states $x$ through the algebraic equations $g$. In (3.13) each trajectory must satisfy the algebraic constraint $g(x, y)=0$ at all time. Therefore, (3.13) can be interpreted as a dynamical system on the algebraic manifold $L=(x, y): g(x, y)=0$. Generally, there exists certain points within $L$ where the trajectories may run into conflicts in satisfying constraint $g(x, y)=0$. These points are called singular or the impasse points of the network.

Given a point on $L$, say $\left(x_{o}, y_{o}\right) \in L$. If the Jacobian $G_{y}=\partial g / \partial y$ is nonsingular (i.e., $\operatorname{det}\left(G_{y}\right) \neq 0$ at $\left(x_{o}, y_{o}\right)$ ), by virtue of the Implicit Function Theorem, there exist a unique solution, $y=h(x)$, of the network equations which satisfies $g(x, h(x))=0$ locally near $\left(x_{o}, y_{o}\right)$. Therefore locally near $\left(x_{o}, y_{o}\right)$, the dynamics of (3.13) exists as a well defined smooth system described by

$$
\dot{x}=f(x, h(x))=f_{o}(x)
$$

which is indeed an ODE system.

The purpose of the application of Lyapunov theory in the multi-machine power system is to obtain an energy function (or Lyapunov function) for (3.27). The following assumptions are considered (some of them already mentioned):

1. The power system is lossless.

2. Generators are modeled by the one-axis model.

3. AVR and turbine regulator are excluded.

4. Damping in the synchronous machine is zero $(D=0)$.

5. Real part of the loads are constant, $m p=0$ (equation(3.8)).

Moreover, the system (3.13) is transformed into the so called Center of Inertia (COI) reference frame. The COI reference frame is defined as follows: 


$$
\begin{gathered}
\delta_{C O I}=\frac{1}{M_{T}} \sum_{k=1}^{n} M_{k} \delta_{k} \\
\omega_{C O I}=\frac{1}{M_{T}} \sum_{k=1}^{n} M_{k} \omega_{k}
\end{gathered}
$$

where

$$
M_{T}=\sum_{k=1}^{n} M_{k}
$$

the motion of the COI is defined by:

$$
\dot{\omega}_{C O I}=\frac{1}{M_{T}} \sum_{k=1}^{n} M_{k} \dot{\omega}_{k}=\frac{1}{M_{T}} \sum_{k=1}^{n}\left(P_{m k}-P_{e k}\right)=\frac{P_{C O I}}{M_{T}}
$$

note that at any equilibrium point, $x_{o}, P_{C O I}=0$. The states variables $\delta_{k}$ and $\omega_{k}$ are transformed to the COI variables as:

$$
\begin{aligned}
\tilde{\delta}_{k} & =\delta_{k}-\delta_{C O I} \\
\tilde{\omega}_{k} & =\omega_{k}-\omega_{C O I}
\end{aligned}
$$

these COI variables are constrained by:

$$
\begin{aligned}
& \sum_{k=1}^{n} M_{k} \tilde{\delta}_{k}=0 \\
& \sum_{k=1}^{n} M_{k} \tilde{\omega}_{k}=0
\end{aligned}
$$

The load buses angles are also transformed to the COI reference frame by

$$
\tilde{\theta}_{k}=\theta_{k}-\delta_{C O I}
$$

the time derivative of equation (3.28) gives: 


$$
\dot{\tilde{\delta}}_{k}=\dot{\delta}_{k}-\dot{\delta}_{C O I}=\omega_{k}-\omega_{C O I}=\tilde{\omega}_{k}
$$

and

$$
\dot{\tilde{\omega}}_{k}=\dot{\omega}_{k}-\dot{\omega}_{C O I}=\frac{1}{M_{k}}\left(P_{m k}-P_{e k}-\frac{M_{k}}{M_{T}} P_{C O I}\right)
$$

Thus, the system (3.13) in the COI reference frame is expressed as (for $k=1 \ldots n)$

$$
\begin{aligned}
& \dot{\tilde{\delta}}_{k}=\tilde{\omega}_{k} \\
& \dot{\tilde{\omega}}_{k}=\frac{1}{M_{k}}\left(P_{m k}-\frac{E_{q k} U_{k}}{x_{d k}^{\prime}} \sin \left(\tilde{\delta}_{k}-\tilde{\theta}_{k}\right)-\frac{M_{k}}{M_{T}} P_{C O I}\right) \\
& \dot{E}_{q}^{\prime}=\frac{1}{T_{d o k}^{\prime}}\left(E_{f k}-\frac{x_{d k}}{x_{d k}^{\prime}} E_{q k}^{\prime}+\frac{x_{d k}-x_{d k}^{\prime}}{x_{d k}^{\prime}} U_{k} \cos \left(\tilde{\delta}_{k}-\tilde{\theta}_{k}\right)\right)
\end{aligned}
$$

and

$$
\begin{aligned}
& 0=P_{k}+P_{L k} \\
& 0=Q_{k}+Q_{L k}
\end{aligned}
$$

where $P_{k}$ and $Q_{k}$ are given by equation (3.10) and equation (3.11) in which $\theta$ and $\delta$ are replaced by $\tilde{\theta}$ and $\tilde{\delta}$. Note however that $\tilde{\theta}_{k}-\tilde{\theta}_{l}=\theta_{k}-\theta_{l}$ and $\tilde{\delta}_{k}-\tilde{\delta}_{l}=\delta_{k}-\delta_{l}$.

Based on the mentioned assumptions and the COI reference frame, the following energy function is given for the DAE (3.29) [35].

$$
\mathcal{V}\left(\tilde{\omega}, \tilde{\delta}, E_{q}^{\prime}, U, \tilde{\theta}\right)=\mathcal{V}_{1}+\sum_{k=1}^{7} \mathcal{V}_{2 k}+C_{o}
$$

where:

$$
\begin{aligned}
\mathcal{V}_{1} & =\frac{1}{2} \sum_{k=1}^{n} M_{k} \tilde{\omega}_{k}^{2} \\
\mathcal{V}_{21} & =-\sum_{k=1}^{n} P_{m k} \tilde{\delta}_{k} \\
\mathcal{V}_{22} & =\sum_{k=1}^{N} P_{L k} \tilde{\theta}_{k}
\end{aligned}
$$




$$
\begin{aligned}
& \mathcal{V}_{23}=\sum_{k=1}^{N} \int \frac{Q_{L k}}{U_{k}} d U_{k} \\
& \mathcal{V}_{24}=\sum_{k=1}^{n} \frac{1}{2 x_{d k}^{\prime}}\left[E_{q k}^{\prime 2}+U_{k}^{2}-2 E_{q k}^{\prime} U_{k} \cos \left(\tilde{\delta}_{k}-\tilde{\theta}_{k}\right)\right] \\
& \mathcal{V}_{25}=-\frac{1}{2} \sum_{k=1}^{N} \sum_{l=1}^{N} B_{k l} U_{k} U_{l} \cos \left(\tilde{\theta}_{k}-\tilde{\theta}_{l}\right) \\
& \mathcal{V}_{26}=-\sum_{k=1}^{n} \frac{E_{f d k} E_{q k}^{\prime}}{x_{d k}-x_{d k}^{\prime}} \\
& \mathcal{V}_{27}=\sum_{k=1}^{n} \frac{E_{q k}^{\prime 2}}{2\left(x_{d k}-x_{d k}^{\prime}\right)}
\end{aligned}
$$

with:

$\mathcal{V}_{1}$ : total change in the kinetic energy

$\mathcal{V}_{2 k}$ : total change in the potential energy

$C_{o}$ : constant, such that the energy function is zero at the stable equilibrium point

Using the notation $\left[\frac{d \mathcal{V}}{d t}\right]_{\tilde{\omega}}$ for $\frac{\partial \mathcal{V}}{\partial \tilde{\omega}} \frac{d \tilde{\omega}}{d t}$, and similarly for the other states, $\dot{\mathcal{V}}(x)$ becomes [22]:

$$
\begin{aligned}
{\left[\frac{d \mathcal{V}_{1}}{d t}\right]_{\tilde{\omega}}+\left[\frac{d \mathcal{V}_{21}}{d t}+\frac{d \mathcal{V}_{24}}{d t}\right]_{\tilde{\delta}} } & =0 \\
& {\left[\frac{d \mathcal{V}_{22}}{d t}+\frac{d \mathcal{V}_{24}}{d t}+\frac{d \mathcal{V}_{25}}{d t}\right]_{\tilde{\theta}}=\sum\left(P_{k}+P_{L k}\right) \dot{\tilde{\theta}}_{k}=0 } \\
& {\left[\frac{d \mathcal{V}_{23}}{d t}+\frac{d \mathcal{V}_{24}}{d t}+\frac{d \mathcal{V}_{25}}{d t}\right]_{U}=\sum\left(Q_{k}+Q_{L k}\right) \frac{\dot{U}_{k}}{U_{k}}=0 } \\
& {\left[\frac{d \mathcal{V}_{24}}{d t}+\frac{d \mathcal{V}_{26}}{d t}+\frac{d \mathcal{V}_{27}}{d t}\right]_{E_{q}^{\prime}}=-\sum_{k=1}^{n} \frac{T_{d o k}^{\prime}}{x_{d k}-x_{d k}^{\prime}}\left(\dot{E}_{q k}^{\prime}\right)^{2} }
\end{aligned}
$$

Thus, the time derivative of the energy function is: 


$$
\frac{d \mathcal{V}(x)}{d t}=-\sum_{k=1}^{n} \frac{T_{d o k}^{\prime}}{x_{d k}-x_{d k}^{\prime}}\left(\dot{E}_{q k}^{\prime}\right)^{2} \leq 0
$$

which is non-positive.

\subsection{Control Lyapunov Function (CLF)}

CLF basically consists, in the use of Lyapunov function candidates in feedback design itself by making the Lyapunov time derivative negative when choosing the control.

Consider the following autonomous system depending on a parameter $u \in R^{r}$.

$$
\dot{x}=f(x, u) \quad, \quad x \in \mathcal{D} \subset R^{n_{x}}
$$

where $\mathcal{D}$ is an open connected region of $R^{n_{x}}$. System (3.37) is referred to as a control system in which $u$ is the control input and $x$ is the state variables.

Let $x_{o}$ be an equilibrium point for (3.37) and $x_{o} \in \mathcal{D}$. The stabilizability problem can then be stated in the following way. We want to find conditions for the existence for feedback control $u=u(x)$ defined in a neighborhood of the origin such that the closed-loop system

$$
\dot{x}=f(x, u(x))=\tilde{f}(x)
$$

has a stable equilibrium point at $x_{o}$. If such a function $u(x)$ exists, we say that (3.37) is stabilizable at the equilibrium point and the function $u(x)$ is called a stabilizing feedback law or (simply) stabilizer.

Theorem 3 Let $x_{o}$ be an asymptotically stable equilibrium point for the system (3.17) and $\mathcal{D} \subset R^{n_{x}}$ be a domain containing $x_{o}$. Let also $\mathcal{D}_{o} \subset \mathcal{D}$, containing $x_{o}$. Then, there is a continuously differentiable positive definite function $\mathcal{V}: \mathcal{D}_{o} \rightarrow R$ whose time derivative is negative definite.

Consider system (3.37) and assume that this system is continuously stabilizable. According to Theorem 3 (converse Lyapunov theorem) there must be a positive definite function $\mathcal{V}(x)$, such that

$$
\dot{\mathcal{V}}(x)=\nabla \mathcal{V}(x) \cdot f(x, u)<0
$$


for each $x \neq x_{o}$ in some neighborhood $\mathcal{D}$ of the origin [34].

A function $\mathcal{V}(x)$ satisfying (3.18), (3.19) and (3.38) is called a Control Lyapunov Function (CLF). Henceforth, a special form of (3.38) which is called affine systems, is considered. These systems are of the form:

$$
\dot{x}=f(x, u)=f_{o}(x)+\sum_{i=1}^{r} u_{i} f_{i}(x)
$$

where $x \in \mathcal{D} \subset R^{n_{x}}$ and $f_{o}(x)$ is the system without control input. It is assumed that $f_{o}, f_{1}, \ldots, f_{r}$ are $C^{\infty},\left(C^{s}\right.$ denotes $s$-time differentiable).

If there is a $C^{\infty}$ function $\mathcal{V}(x)$ which satisfies (3.18), (3.19) and

$$
\nabla \mathcal{V}(x) \cdot f_{o}(x) \leq 0 \quad \text { for } \quad x \in \mathcal{D}
$$

is said that (3.39) satisfies a Lyapunov function of the Jurdjevic-Quinn type [39]. According to the Jurdjevic-Quinn approach, a stabilizing feedback law is typically defined componentwise, setting $u(x)=\left[u_{1}(x), \ldots, u_{r}(x)\right]$ and

$$
u_{i}(x)=-\nabla \mathcal{V}(x) \cdot f_{i}(x), \quad i=1, \ldots, r
$$

Thus, the time derivative of $\mathcal{V}(x)$ for $x \in \mathcal{D}$ with respect to the closed-loop system is given by

$$
\begin{aligned}
\dot{\mathcal{V}}(x) & =\nabla \mathcal{V}(x) \cdot f_{o}(x)+\sum_{i=1}^{r} u_{i} \nabla \mathcal{V}(x) \cdot f_{i}(x) \\
& \leq \sum_{i=1}^{r} u_{i} \nabla \mathcal{V}(x) \cdot f_{i}(x)=-\sum_{i=1}^{r} u_{i}\left(\nabla \mathcal{V}(x) \cdot f_{i}(x)\right)^{2}<0
\end{aligned}
$$

by virtue of (3.40) and (3.41), [36]. Detailed description about CLF, can be found in [22] and [37] - [39].

Let us illustrate the idea of CLF with a simple example [40]. Consider the following system with control, $\dot{x}=f(x, u)$ :

$$
\begin{aligned}
& \dot{x}_{1}=x_{2} \\
& \dot{x}_{2}=-x_{1}+u
\end{aligned}
$$

or 


$$
\begin{aligned}
\dot{x} & =\left[\begin{array}{cc}
0 & 1 \\
-1 & 0
\end{array}\right]\left[\begin{array}{l}
x_{1} \\
x_{2}
\end{array}\right]+\left[\begin{array}{l}
0 \\
1
\end{array}\right] u \\
& =f_{o}(x)+f_{1} u
\end{aligned}
$$

where $u$ is a stabilizing unknown feedback. Let the following function be a Lyapunov function:

$$
\mathcal{V}(x)=\frac{3}{2} x_{1}^{2}+x_{1} x_{2}+x_{2}^{2}
$$

The time derivative of (3.44) along the trajectories of (3.43) is given by:

$$
\begin{aligned}
& \dot{\mathcal{V}}(x, u)=\nabla \mathcal{V}(x) \cdot f(x, u) \\
& \dot{\mathcal{V}}(x, u)=-x_{1}^{2}+x_{1} x_{2}+x_{2}^{2}-\left(x_{1}+2 x_{2}\right) u
\end{aligned}
$$

This expression is affine in $u$. Thus, if a state such that $x_{1}+2 x_{2} \neq 0$, then we may choose a control value $u$ such that $\dot{\mathcal{V}}<0$. On the other hand, if $x_{1}+2 x_{2}=0$, then the expression reduces to $\dot{\mathcal{V}}=-5 x_{2}^{2}$ (for any $u$ ), which is negative unless $x_{2}$ (and hence also $x_{1}=-2 x_{2}$ ) vanishes. Let us set $u=-x_{2}$, then $\dot{\mathcal{V}}$ becomes:

$$
\dot{\mathcal{V}}(x, u)=-x_{1}^{2}-x_{2}^{2}<0
$$

The choice $u=-x_{2}$ has made $\dot{\mathcal{V}}(x, u)$ to become negative definite and the system (3.43) to be asymptotically stable. In conclusion, for each $x \neq 0$ there is some $u$ so that $\dot{\mathcal{V}}(x, u)<0,[40]$.

\subsection{Modal Analysis}

Consider the linearized form of (3.13):

$$
\begin{aligned}
\Delta \dot{x} & =A \Delta x(t)+B \Delta u \\
\Delta \mathcal{Y} & =C \Delta x+D \Delta u
\end{aligned}
$$

where:

$\Delta \dot{x}$ is the state vector (dimension $\left.n_{x} \times 1\right)$ 
$\Delta u$ is the input vector (dimension $r \times 1$ )

$\Delta \mathcal{Y}$ is the output vector (dimension $m \times 1$ )

$A$ is the state matrix (dimension $n_{x} \times n_{x}$ )

$B$ is the input matrix (dimension $n_{x} \times r$ )

$C$ is the output matrix (dimension $m \times n_{x}$ )

$D$ is the (feedforward) matrix which defines the proportion of input which appears directly in the output (dimension $m \times r$ )

The eigenvalues of matrix $A$ are given by

$$
\operatorname{det}(A-\lambda \mathbf{I})=0
$$

Expansion of the determinant gives the characteristic equation. The $n_{x}$ solutions of $\lambda=\lambda_{1}, \ldots, \lambda_{n_{x}}$ are eigenvalues of $A$.

Any non-zero vector $V_{i}^{r}$ which satisfies

$$
A V_{i}^{r}=\lambda_{i} V_{i}^{r}
$$

is termed the right eigenvector of $A$, corresponding to the eigenvalue $\lambda_{i} . V_{i}^{r}$ has dimension $n_{x} \times 1$. The $n_{x}$ right eigenvectors conform the modal matrix $V^{R}$ :

$$
V^{R}=\left[\begin{array}{llll}
V_{1}^{r} V_{2}^{r} & \ldots & V_{n_{x}}^{r}
\end{array}\right]_{n_{x} \times n_{x}}
$$

Likewise, any non-zero vector $V_{i}^{l}$ which satisfies

$$
V_{i}^{l} A=\lambda_{i} V_{i}^{l}
$$

is termed the left eigenvector of $A$ corresponding to the eigenvalue $\lambda_{i}$. $V_{i}^{l}$ has dimension $1 \times n_{x}$. The $n_{x}$ left eigenvectors conform the modal matrix $V^{L}$ :

$$
V^{L}=\left[\left(V_{1}^{l}\right)^{T}\left(V_{2}^{l}\right)^{T} \ldots\left(V_{n_{x}}^{l}\right)^{T}\right]_{n_{x} \times n_{x}}^{T}
$$

The $n_{x}$ eigenvalues can also be expressed in the following matrix form 


$$
\Lambda=\left[\begin{array}{cccc}
\lambda_{1} & 0 & \ldots & 0 \\
0 & \lambda_{2} & \ldots & 0 \\
\vdots & \vdots & \ddots & \vdots \\
0 & 0 & \ldots & \lambda_{n_{x}}
\end{array}\right]_{n_{x} \times n_{x}}
$$

In terms of the modal matrices, equation (3.46) can also be expressed as

$$
\begin{aligned}
A V^{R} & =V^{R} \Lambda \\
\left(V^{R}\right)^{-1} A V^{R} & =\left(V^{R}\right)^{-1} V^{R} \Lambda=\Lambda
\end{aligned}
$$

Thus, the matrix $A$ can be diagonalized by its modal matrix $V^{R}$

By using the modal matrices, the power system described by equation (3.45) can be transformed into a dynamic system of $n_{x}$ uncoupled first order differential equations. Let us define a new state vector, $\xi$, as:

$$
\xi=V^{L} \Delta x=\left(V^{R}\right)^{-1} \Delta x
$$

which implies that

$$
\Delta x=V^{R} \xi
$$

Equation (3.51) is substituted in the linearized system (3.45)

$$
\begin{aligned}
V^{R} \dot{\xi} & =A V^{R} \xi+B \Delta u \\
\Delta \mathcal{Y} & =C V^{R} \xi+D \Delta u
\end{aligned}
$$

which results in the following $n_{x}$-uncoupled first-order differential algebraic system

$$
\begin{aligned}
\dot{\xi} & =\Lambda \xi+V^{L} B \Delta u \\
\Delta \mathcal{Y} & =C V^{R} \xi+D \Delta u
\end{aligned}
$$

where

$$
\Lambda=\left(V^{R}\right)^{-1} A V^{R} \text { by virtue of }(3.49)
$$


If we assume that $\mathcal{Y}$ is not a direct function of $u$ (i.e., $D=0$ ), the application of Laplace transform in system (3.52) results in:

$$
\begin{aligned}
\xi(s) & =(s \mathbf{I}-\Lambda)^{-1} V^{L} B \Delta u(s) \\
\Delta \mathcal{Y}(s) & =C V^{R}(s \mathbf{I}-\Lambda)^{-1} V^{L} B \Delta u(s)
\end{aligned}
$$

the transfer function of the system (3.53) is:

$$
\begin{aligned}
G(s) & =\frac{\Delta \mathcal{Y}}{\Delta u(s)} \\
G(s) & =C V^{R}(s \mathbf{I}-\Lambda)^{-1} V^{L} B
\end{aligned}
$$

since $\Lambda$ is a diagonal matrix, the transfer function can be expressed in partial functions as:

$$
G(s)=\sum_{i=1}^{n_{x}} \frac{R_{i}}{s-\lambda_{i}}
$$

where $R_{i}$, of order $m \times r$, is the residue of $G(s)$ at $\lambda_{i}$ and is defined as:

$$
R_{i}=C V_{i}^{r} V_{i}^{l} B
$$

By having a feedback transfer function of the form $H(s, k)=k H(s)$, where $k$ is a constant gain between output and input, it can be shown that (with $s=\lambda_{i}$ ) [41]:

$$
\frac{\partial \lambda_{i}}{\partial k}=R_{i} H\left(\lambda_{i}\right)
$$

for small values of gain, equation (3.56) can be written as:

$$
\frac{\Delta \lambda_{i}}{\Delta k}=R_{i} H\left(\lambda_{i}\right)
$$

If a feedback transfer function is added to the open system (3.53), it can be shown that [41]

$$
\begin{array}{r}
\Delta \lambda_{i}=R_{i} H\left(\lambda_{i}, k\right) \\
\Delta \lambda_{i}=k R_{i} H\left(\lambda_{i}\right)
\end{array}
$$


Equation (3.58) implies that the position of a selected eigenvalue (or mode) can be changed by tuning the parameters of the transfer function $H\left(s=\lambda_{i}\right)$ based on the information from the residue $R_{i}$.

\subsubsection{Tuning of Regulator}

The purpose of the regulator is to add Power Oscillation Damping (POD) to the generator rotor oscillations by modulating the active power through the VSC-HVdc. Figure 3.3 shows the feedback control system.

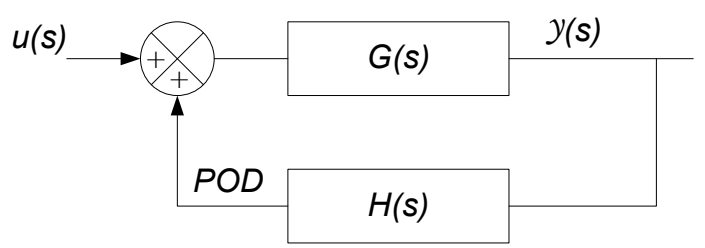

Figure 3.3: Feedback control system

The open loop transfer function $G(s)$ is determined by equation (3.54). $H(s)$ is the transfer function of the regulator whose output is the Power Oscillation Damping (POD) signal. The regulator consists of three blocks: a wash-out filter, a phase compensating filter and a gain. The regulator is shown in Figure 3.4.

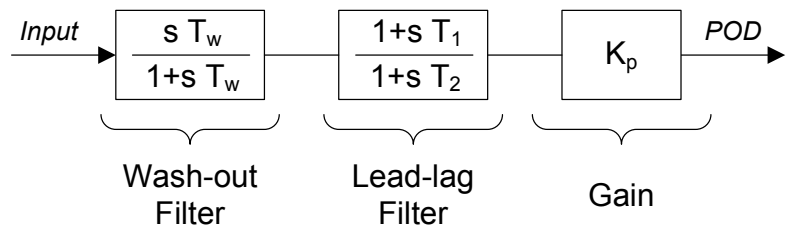

Figure 3.4: Damping Regulator

The wash-out filter serves as a high-pass filter, with the time constant $T_{w}$ high enough to allow signals associated with oscillations to pass unchanged. The value of $T_{w}$ is not critical and may be in the range of 1 to 20 seconds [42].

The phase compensation block provides the appropriate phase-lead or phase-lag characteristic. For practical reasons, the argument of the phase compensation is limited to a maximum of $60^{\circ}$ [42]. This is the reason why 
in practice two or more first-order blocks may be used to achieve the desired compensation.

The stabilizer gain determines the amount of damping introduced by the regulator. Ideally, the gain should be set at a value corresponding to maximum damping; however, it is often limited by other considerations.

The residue of the oscillatory mode of interest, which gives the direction of the eigenvalue departure for a small change of the system parameters, is given by equation (3.55). In order to move the eigenvalue $\lambda_{i}$ to a desired position (only the real part is changed) the argument of $H\left(s=\lambda_{i}\right), \phi$, must be $\phi=180^{\circ}-\arg \left(R_{i}\right)$; see Figure 3.5.

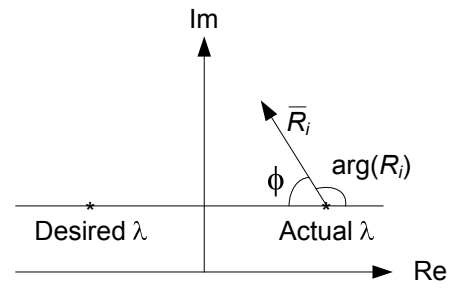

Figure 3.5: Direction of the eigenvalue departure

The transfer function of the lead-lag filter is of the form:

$$
\left(\frac{1+s a T}{1+s T}\right)
$$

where,

$$
\begin{aligned}
& T_{1}=a T \\
& T_{2}=T
\end{aligned}
$$

where $a>1$. To minimize high frequency gain, which amplifies the signal noise level, the parameter $a$ should be as small as possible. The maximum phase lead angle, $\alpha_{\max }$, obtained with this filter is given by the expression [43]:

$$
\alpha_{\max }=\arcsin \left(\frac{a-1}{a+1}\right)
$$

In our case, $\alpha_{\max }$ is the desired argument of the transfer function of the regulator, $\phi$. Thus, 


$$
\phi=\arcsin \left(\frac{a-1}{a+1}\right)
$$

and the parameter $a$ can now be calculated

$$
a=\frac{1+\sin (\phi)}{1-\sin (\phi)}
$$

The time constant $T$ determines the frequency $\omega_{\max }$ at which this maximum phase lead angle occurs:

$$
\omega_{\max }=\frac{1}{T \sqrt{a}}
$$

The constant $T$ is chosen so as to make this maximum occurs at the frequency of the electromechanical mode. The mode of our interest is $\lambda_{i}=\sigma_{i} \pm j \omega_{i}$. Therefore $\omega_{\max }=\omega_{i}$ and the constant $T$ is

$$
T=\frac{1}{\omega_{i} \sqrt{a}}
$$

Thereby, the time constants of the lead-lag filter $T_{1}$ and $T_{2}$ can be calculated by using equation (3.59). 



\section{Chapter 4}

\section{Control Strategies for a VSC-HVdc}

The derivation of the control strategies are based on two different theoretical frames: Nonlinear theory and Linear theory.

Nonlinear theory is considered in the derivation of the control strategy to improve the transient stability of the system. More specifically, the control strategy is based on Control Lyapunov Function theory.

Linear theory or small signal analysis is used in the derivation of the control strategies to improve damping in the system. The derivation of this strategy is based on the information obtained from the modal matrices.

Although each control strategy is intended for a specific purpose, i.e., CLF for transient stability and modal analysis for damping, the CLF-based strategy can also improve the damping of the system and modal-analysisbased strategy may also enhance the transient stability of the system.

\subsection{Control Lyapunov Function}

Consider the system described by equation (3.29) and equation (3.30). The assumptions that resulted in this set of DAE are still valid. This system is referred to as the uncontrolled system. For this uncontrolled system, the energy function (or Lyapunov function) (3.31) is proposed. The time derivative of this energy function along the trajectories of the uncontrolled system is given by (3.36), which is non-positive. Let us denote this time derivative as $\dot{\mathcal{V}}_{\text {uncontrolled, }}$ 


$$
\dot{\mathcal{V}}_{\text {uncontrolled }}=-\sum_{k=1}^{n} \frac{T_{d o k}^{\prime}}{x_{d k}-x_{d k}^{\prime}}\left(\dot{E}_{q k}^{\prime}\right)^{2}
$$

Let us now connect an uncontrolled VSC-HVdc in the system. Uncontrolled VSC-HVdc means that the modulated (or control) variables in equation (2.7) are not considered, i.e., $\Delta E_{i}^{r e}=\Delta E_{i}^{i m}=\Delta E_{j}^{r e}=\Delta E_{j}^{i m}=0$. Due to the inclusion of the uncontrolled VSC-HVdc it is necessary to find a new energy function for the power system. By following the procedure described in [44] the construction of the new energy function results in:

$$
\mathcal{V}_{\text {uncontrolled }}^{H V d c}=\mathcal{V}_{1}+\sum_{k=1}^{7} \mathcal{V}_{2 k}+\mathcal{V}_{H V d c_{o}}+C_{o}
$$

where

$$
\begin{aligned}
\mathcal{V}_{H V d c_{o}} & =\frac{b_{t i}}{2}\left[U_{i}^{2}-2 U_{i}\left(\cos \tilde{\theta}_{i} E_{i o}^{r e}+\sin \tilde{\theta}_{i} E_{i o}^{i m}\right)\right] \\
& +\frac{b_{t j}}{2}\left[U_{j}^{2}-2 U_{j}\left(\cos \tilde{\theta}_{j} E_{j o}^{r e}+\sin \tilde{\theta}_{j} E_{j o}^{i m}\right)\right]
\end{aligned}
$$

The inclusion of the uncontrolled VSC-HVdc in the power systems results in the additional term $\mathcal{V}_{H V d c_{o}}$ in the energy function (3.31). However, this new term does not alter the negativeness of (4.1), since at bus $i$ and bus $j$, $\dot{\mathcal{V}}_{\text {uncontrolled }}^{H V \text { is given by }}$

$$
\begin{array}{r}
{\left[\frac{d \mathcal{V}_{22}}{d t}+\frac{d \mathcal{V}_{24}}{d t}+\frac{d \mathcal{V}_{25}}{d t}+\frac{d \mathcal{V}_{H V d c_{o}}}{d t}\right]_{\tilde{\theta}_{i}}} \\
=\left(P_{i}+P_{L i}+P_{s i o}\right) \dot{\tilde{\theta}}_{i}=0 \\
{\left[\frac{d \mathcal{V}_{23}}{d t}+\frac{d \mathcal{V}_{24}}{d t}+\frac{d \mathcal{V}_{25}}{d t}+\frac{d \mathcal{V}_{H V d c_{o}}}{d t}\right]_{U_{i}}} \\
=\left(Q_{i}+Q_{L i}+Q_{s i o}\right) \frac{\dot{U}_{i}}{U_{i}}=0
\end{array}
$$




$$
\begin{array}{r}
{\left[\frac{d \mathcal{V}_{22}}{d t}+\frac{d \mathcal{V}_{24}}{d t}+\frac{d \mathcal{V}_{25}}{d t}+\frac{d \mathcal{V}_{H V d c_{o}}}{d t}\right]_{\tilde{\theta}_{j}}} \\
=\left(P_{j}+P_{L j}+P_{\text {sjo }}+P_{\text {losses }}\right) \dot{\tilde{\theta}}_{j}=0 \\
{\left[\frac{d \mathcal{V}_{23}}{d t}+\frac{d \mathcal{V}_{24}}{d t}+\frac{d \mathcal{V}_{25}}{d t}+\frac{d \mathcal{V}_{H V d c_{o}}}{d t}\right]_{U_{j}}} \\
=\left(Q_{j}+Q_{L j}+Q_{\text {sjo }}\right) \frac{\dot{U}_{j}}{U_{j}}=0
\end{array}
$$

Thus,

$$
\dot{\mathcal{V}}_{\text {uncontrolled }}^{H V d c}=\dot{\mathcal{V}}_{\text {uncontrolled }}
$$

Now let us consider the modulated parts of the VSC-HVdc. This implies that $E_{i}^{r e}, E_{i}^{i m}, E_{j}^{r e}$ and $E_{j}^{i m}$ are not fixed anymore, since $\left(\Delta E_{i}^{r e}, \Delta E_{i}^{i m}, \Delta E_{j}^{r e}, \Delta E_{j}^{i m}\right) \neq 0$. Thus, with modulated (or control) variables we are dealing with a controlled system similar to (3.39). Now using the energy function (4.2) for the controlled system, the time derivative of this function along the trajectories of this controlled system is given by:

$$
\dot{\mathcal{V}}(x)=\dot{\mathcal{V}}_{\text {uncontrolled }}^{H V d c}+\dot{\mathcal{V}}_{\text {controlled }}^{H V d c}
$$

where $\dot{\mathcal{V}}_{\text {uncontrolled }}^{H V d c}$ is defined by equation (4.6).

When the VSC-HVdc is controlled, the time derivative of the energy function of the uncontrolled system, equation (4.1), is affected, since equations (3.33) and equation (3.34) are not zero anymore. In order to see the changes, consider the $i$-th term in equation (3.33):

$$
\left(P_{i}+P_{L i}\right) \dot{\tilde{\theta}}_{i}
$$

Without a VSC-HVdc connected to bus $i,\left(P_{i}+P_{L i}\right)=0$, which results in the zero summation in equation (3.33). When a VSC-HVdc is connected at bus $i$, the power balance gives $P_{i}+P_{L i}+P_{\text {sio }}+\Delta P_{s i}=0$. Therefore, the $i$-th term in equation (3.33) becomes:

$$
\left(P_{i}+P_{L i}+P_{s i o}\right) \dot{\tilde{\theta}}_{i}=-\Delta P_{s i} \dot{\tilde{\theta}}_{i}
$$

By applying same considerations to the second bus of connection of the HVdc link, i.e., bus $j$, equation (3.33) results in: 


$$
\begin{aligned}
& {\left[\frac{d \mathcal{V}_{22}}{d t}+\frac{d \mathcal{V}_{24}}{d t}+\frac{d \mathcal{V}_{25}}{d t}\right]_{\tilde{\theta}}} \\
& \quad=-\Delta P_{s i} \dot{\tilde{\theta}}_{i}-\Delta P_{s j} \dot{\tilde{\theta_{j}}}
\end{aligned}
$$

Concerning the reactive power, the corresponding terms in equation (3.34) results in:

$$
\begin{aligned}
& {\left[\frac{d \mathcal{V}_{23}}{d t}+\frac{d \mathcal{V}_{24}}{d t}+\frac{d \mathcal{V}_{25}}{d t}\right]_{U}} \\
& =-\Delta Q_{s i} \frac{\dot{U}_{i}}{U_{i}}-\Delta Q_{s j} \frac{\dot{U}_{j}}{U_{j}}
\end{aligned}
$$

Therefore, the time derivative of the controlled system, $\dot{\mathcal{V}}_{\text {controlled }}^{H V d c}$ becomes:

$$
\dot{\mathcal{V}}_{\text {controlled }}^{H V d c}=-\Delta P_{s i} \dot{\tilde{\theta}}_{i}-\Delta P_{s j} \dot{\tilde{\theta}}_{j}-\Delta Q_{s i} \frac{\dot{U}_{i}}{U_{i}}-\Delta Q_{s j} \frac{\dot{U}_{j}}{U_{j}}
$$

The objective of Control Lyapunov Function is to keep $\dot{\mathcal{V}}_{\text {controlled }}^{H V d c}$ non-positive; therefore the following definition and criterion are adopted:

1. $\Delta P_{s j}=-\Delta P_{s i}$ which follows $(2.10)$

2. $\Delta Q_{s i}=\Delta Q_{s j}=0$, i.e., only active power is controlled

Thus, equation (4.9) becomes:

$$
\begin{aligned}
& \dot{\mathcal{V}}_{\text {controlled }}^{H V d c}=-\Delta P_{s i} \dot{\tilde{\theta}}_{i}+\Delta P_{\text {si }} \dot{\tilde{\theta}}_{j}+0+0 \\
& \dot{\mathcal{V}}_{\text {controlled }}^{H V d c}=-\Delta P_{s i}\left(\dot{\tilde{\theta}}_{i}-\dot{\tilde{\theta}}_{j}\right)
\end{aligned}
$$

$\dot{\mathcal{V}}_{\text {controlled }}^{H V d c}$ becomes non-positive if:

$$
\begin{aligned}
\Delta P_{s i} & =k\left(\dot{\tilde{\theta}}_{i}-\dot{\tilde{\theta}}_{j}\right) \\
& =k f_{i j}
\end{aligned}
$$

where: 
$k$ : positive gain

$f_{i j}$ : difference of the frequency of the buses where the VSC-HVdc is connected

Since

$$
\begin{aligned}
\Delta Q_{s i} & =\Delta Q_{s j}=0 \\
\Delta P_{s i} & =k f_{i j} \\
\Delta P_{s j} & =-\Delta P_{s i}=-k f_{i j}
\end{aligned}
$$

solving for $\Delta E_{i}^{r e}, \Delta E_{i}^{i m} \Delta E_{j}^{r e}$ and $\Delta E_{j}^{i m}$ results in:

$$
\begin{array}{rlrl}
\Delta E_{i}^{r e} & =k f_{i j} \frac{U_{i} \sin \tilde{\theta}_{i}}{U_{i}^{2}} & \Delta E_{i}^{i m} & =-k f_{i j} \frac{U_{i} \cos \tilde{\theta}_{i}}{U_{i}^{2}} \\
\Delta E_{j}^{r e} & =-k f_{i j} \frac{U_{j} \sin \tilde{\theta}_{j}}{U_{j}^{2}} & \Delta E_{j}^{i m}=k f_{i j} \frac{U_{j} \cos \tilde{\theta}_{j}}{U_{j}^{2}}
\end{array}
$$

Substituting (4.11) in equation (2.7) and based on equation (2.6), the control variables $E_{i}, E_{j}, \gamma_{i}$ and $\gamma_{j}$ can be determined.

\subsection{Modal Analysis}

Modal analysis is based on the linearized form (3.45) of the system (3.13). The main purpose of the modal analysis is to obtain information about the inherent dynamics characteristics of a power system, such as identification of the different modes, frequency of oscillation of the oscillatory modes and their respective damping ratios. From modal analysis it is also possible to obtain the information to tune the regulator that feedbacks the system to improve the power oscillation damping.

Since one of the main capabilities of a VSC-HVdc is the independent control of active and reactive power, the modulation of both powers is considered for increasing damping. The modulation of the active power is the main selection to improve damping. However, the modulation of the reactive power can be a practical way to increase damping in the system when it is not possible to transfer active power through the HVdc-link, but the converter stations are available. The feedback loop and regulator type described in section 3.5.1 are used for both alternatives. 


\subsubsection{Active Power Control}

Two different signals are considered as input for the regulator. One signal is the magnitude of a line current. The other signal is the difference of the frequencies measured at two buses in the network. Figure 4.1 shows the modal-analysis-based modulation of active power to improve the system stability and damping.

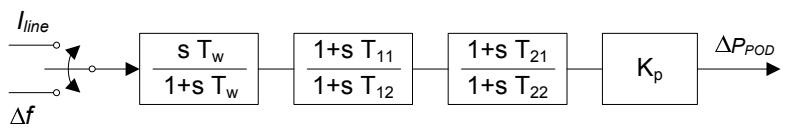

Figure 4.1: Active power control

The matrices $A, B, C$ and $D$ of the linearized power system are obtained directly from the software of simulation [45]. From matrix $A$, the eigenvalues of the system are computed, equation (3.46). The mode of interest, $\lambda_{i}$, is the oscillatory mode with the lowest damping ratio. Once $\lambda_{i}$ is determined, the respective right eigenvector $V_{i}^{r}$ and left eigenvector $V_{i}^{l}$ are computed (equation (3.47) and equation (3.48), respectively). With all this information available, the residue of the mode $i$ is calculated by using equation (3.55).

Next the tuning of the regulator is computed by using the residue method described in section 3.5.1. The calculated argument of the regulator, $\phi=$ $180^{\circ}-\arg \left(R_{i}\right)$, is split into two lead-lag filters. The time constant of the wash out filter is set to $15 \mathrm{~s}$. The gain of the regulator is obtained from Nyquist diagrams.

\subsubsection{Reactive Power Control}

The control algorithm to modulate the reactive power is divided into an Automatic Voltage Regulator (AVR), and a supplementary control to improve the power oscillations damping (POD). The supplementary control is more feasible when the dc- link cannot transmit active power, but the converters are in functions to exchange reactive power. The derivation of the POD signal follows the same procedure of the modulation of active power. The input of the regulator is also the magnitude of a line current. Figure 4.2 shows the modal-analysis-based modulation of reactive power to provide voltage support and improve damping. 


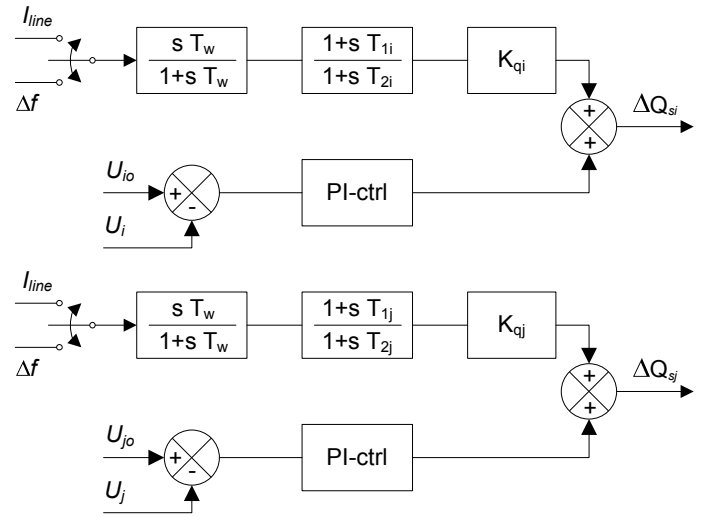

Figure 4.2: Reactive power control

\subsubsection{Modulative Part of the Controllable Variables}

With $\Delta P_{s i}, \Delta Q_{s i}$ and $\Delta Q_{s j}$ known, solving for $\Delta E_{i}^{r e}, \Delta E_{i}^{i m} \Delta E_{j}^{r e}$ and $\Delta E_{j}^{i m}$ results in:

$$
\begin{aligned}
\Delta E_{i}^{r e} & =\frac{U_{i} \sin \theta_{i} \Delta P_{s i}+U_{i} \cos \theta_{i} \Delta Q_{s i}}{b_{t i} U_{i}^{2}} \\
& =\frac{U_{i m i} \Delta P_{s i}+U_{r e i} \Delta Q_{s i}}{b_{t i} U_{i}^{2}} \\
\Delta E_{i}^{i m} & =\frac{-U_{i} \cos \theta_{i} \Delta P_{s i}+U_{i} \sin \theta_{i} \Delta Q_{s i}}{b_{t i} U_{i}^{2}} \\
& =\frac{-U_{r e i} \Delta P_{s i}+U_{i m i} \Delta Q_{s i}}{b_{t i} U_{i}^{2}} \\
\Delta E_{j}^{r e} & =\frac{-U_{j} \sin \theta_{j} \Delta P_{s i}+U_{j} \cos \theta_{j} \Delta Q_{s j}}{b_{t j} U_{j}^{2}} \\
& =\frac{-U_{i m j} \Delta P_{s i}+U_{r e j} \Delta Q_{s j}}{b_{t j} U_{j}^{2}} \\
\Delta E_{j}^{i m} & =\frac{U_{j} \cos \theta_{j} \Delta P_{s i}+U_{j} \sin \theta_{j} \Delta Q_{s j}}{b_{t j} U_{j}^{2}} \\
& =\frac{U_{r e j} \Delta P_{s i}+U_{i m j} \Delta Q_{s j}}{b_{t j} U_{j}^{2}}
\end{aligned}
$$


Substituting (4.12) in equation (2.7) and based on equation (2.6), the control variables $E_{i}, E_{j}, \gamma_{i}$ and $\gamma_{j}$ can be determined.

\subsection{Multichoice Control Strategy}

The derived control strategies become the inputs of the Multichoice Control Strategy of the VSC-HVdc. Figure 4.3 shows the control scheme. Three main inputs feed the Multichoice Control. The first input modulates the active power and groups the signal for transient stability (CLF), power oscillation damping (modal analysis) and power flow. The other two inputs modulate the reactive power (voltage support and modal analysis) in each converter.

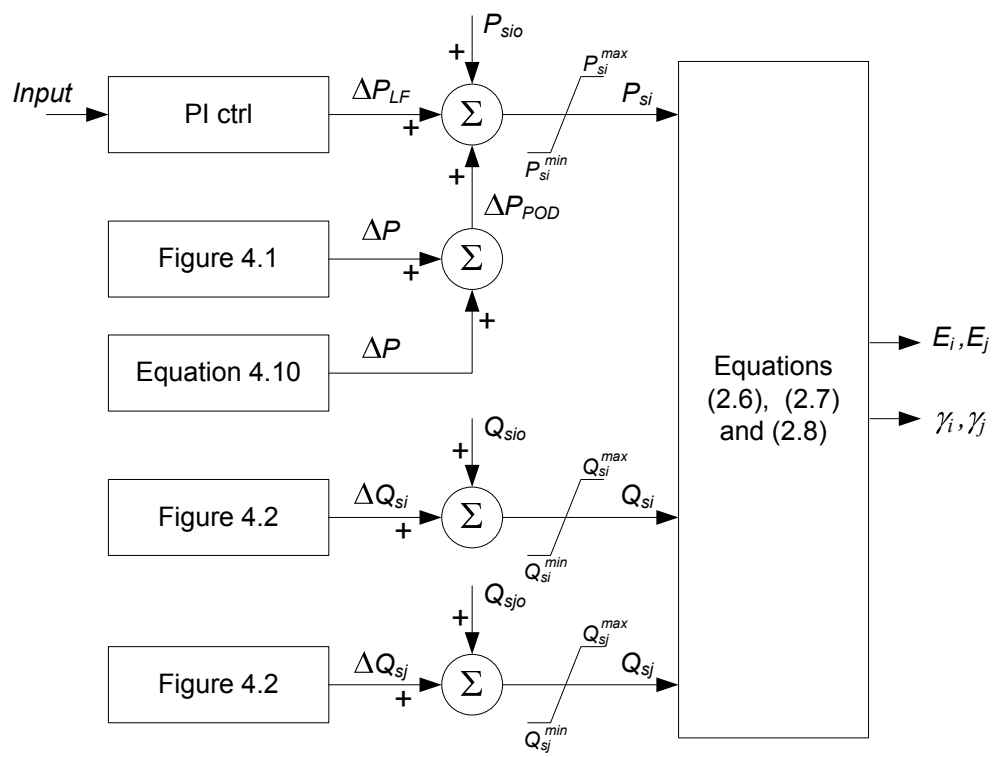

Figure 4.3: Multichoice Control Strategy

The outputs of the Multichoice Control Strategy are the controllable variables (magnitude and angle) of the controllable voltage sources that represent the VSC-HVdc. 


\section{Chapter 5}

\section{Numerical Examples}

\subsection{Test Power System}

The well-known test system shown in Figure 5.1 is used to study the impact of a VSC-HVdc on the system stability and damping. The system data can be found in [42]. The power system has the following modification: Generation at bus 4 is divided into two identical generators. The generators are modeled with one field winding, one damper winding in d-axis and two damper windings in q-axis. The active and reactive components of the loads have constant current and constant impedance characteristics, respectively. The generators are equipped with AVRs, PSSs and turbine governors. Base values are: $S_{b}=100$ MVA and $U_{b}=230 \mathrm{kV}$. All simulations are performed by using SIMPOW [45] and the results are plotted in MATLAB.

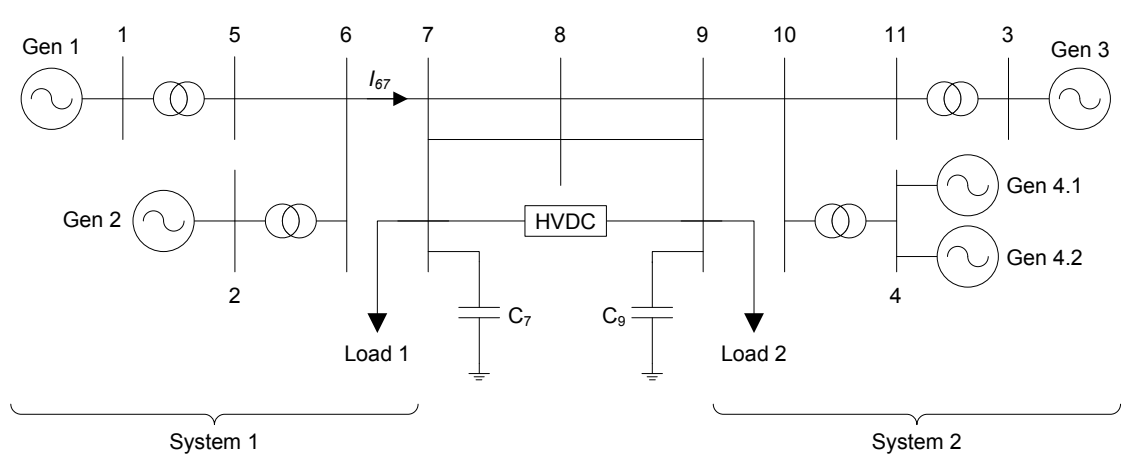

Figure 5.1: Test Power System 
Concerning the VSC-HVdc, the capacity of each converter is $S_{n}=300$ MVA. The active and reactive power limits are based on the MVAcircle, $S_{n}^{2}=P^{2}+Q^{2}$. The restrictions in the circle due to dc-voltage limit or dc-cable are neglected. It is important to note that priority is always given to active power. The losses of the converter stations $P_{\text {losses }}$ (Figure 2.5), are not shown in Figure 5.1, but they are considered in the simulations. $P_{\text {losses }}$ are modeled as constant active power as described in section 2.2.

The following types of system loading are considered:

System loading 1: The total transfer of power from System 1 to System 2 is $350 \mathrm{MW}$.

System loading 2: The total transfer of power from System 1 to System 2 is $450 \mathrm{MW}$. The frequency control in generators $3,4.1$ and 4.2 is blocked and PSSs are disabled in all synchronous machines.

System loading 3: The total transfer of power from System 1 to System 2 is $715 \mathrm{MW}$.

System loading 4: The total transfer of power from System 1 to System 2 is $750 \mathrm{MW}$.

In all four loading systems the initial condition of the voltages at all buses is close to 1 p.u. The transfer of power through the dc-link is $160 \mathrm{MW}$. Also four disturbances are considered in the numerical examples:

Disturbance 1: Temporarily (100 ms) disconnection of $10 \%$ of the load at bus 9 .

Disturbance 2: Three phase short circuit at bus 8 . The fault is cleared $100 \mathrm{~ms}$ later

Disturbance 3: Three phase short circuit at bus 8 . The fault is cleared $100 \mathrm{~ms}$ later by opening one of the lines between bus 8 and bus 9 .

Disturbance 4: Permanent disconnection of generator 4.1 (loss of $350 \mathrm{MW}$ ).

Disturbance 5: Permanent disconnection of partial shunt capacitance at bus 7 (100 Mvar of 300 Mvar). 
Disturbance 6: Permanent interruption of power flow through the dc-link $(160 \mathrm{MW})$.

\subsection{Overall Control of the VSC-HVdc}

The overall control scheme of the VSC-HVdc used in the numerical examples is shown in Figure 5.2. Besides the modulation of active and reactive power to improve the stability of the power system, a load flow control $\left(\Delta P_{L F}\right)$ is also included in the control scheme.

The input signals of the modal-analysis-based active power control, Figure 4.1, is either $\Delta f=f_{7}-f_{9}=f_{79}$, or the magnitude of the current through the line between bus 6 and bus $7, I_{67}$. The supplementary control for the modulation of reactive power, Figure 4.2, also considers $I_{67}$ as the input signal. This supplementary control is used when there is no transfer of active power through the dc-link.

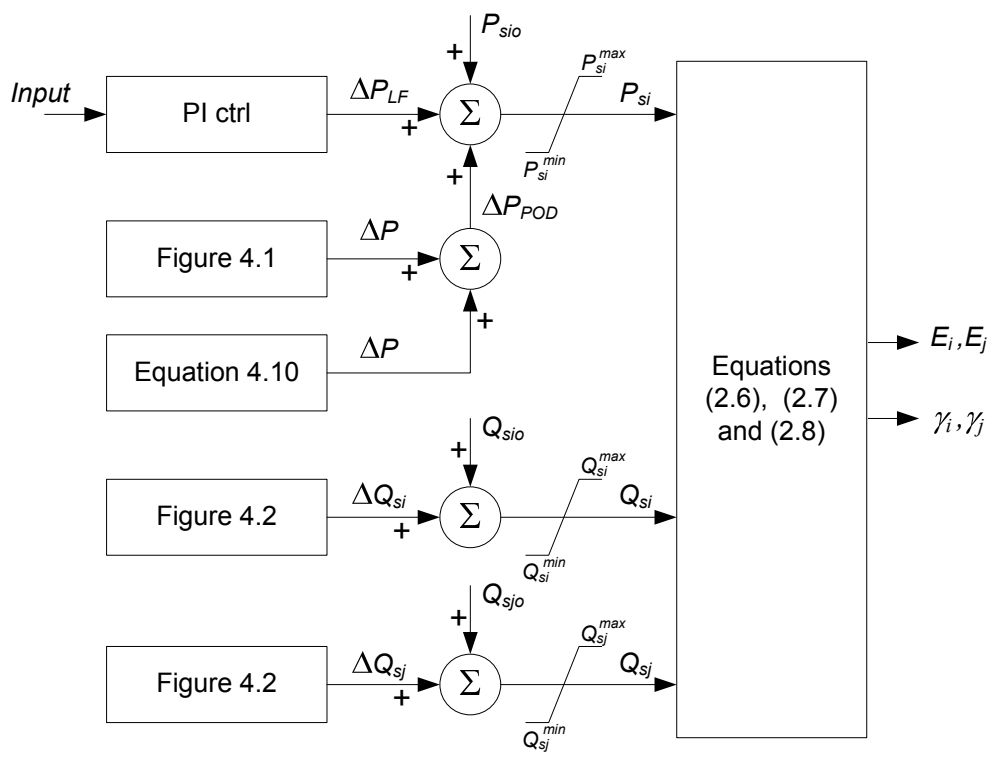

Figure 5.2: Proposed Scheme Control of the VSC-HVdc

In the numerical examples,

Only Active Power Modulation means that $\triangle P_{P O D}$ is modulated by ei- 
ther the modal-analysis-based signal (Figure 4.1) or the CLF-based signal (equation (4.10)) or both. There is no modulation of reactive power from AVRs or modal-analysis-based signal, i.e., $\Delta Q_{s i}=\Delta Q_{s j}=0$ (Figure 4.2). Furthermore, $Q_{s i}=Q_{s i o}$ and $Q_{s j}=Q_{s j o}$.

Active Power Modulation and AVR means that $\triangle P_{P O D}$ is modulated by either the modal-analysis-based signal or the CLF-based signal or both. $\Delta Q_{s i}$ and $\Delta Q_{s j}$ are only modulated by the respective signal coming from AVRs (i.e., $K_{p i}=K_{q i}=0$ ). The reference voltage is the initial voltage magnitude at the buses of connection.

Only Reactive Power Modulation means that $\Delta Q_{s i}$ and $\Delta Q_{s j}$ are modulated by either the modal-analysis-based signal or signal from AVRs or both. Moreover, $\triangle P_{P O D}=0$ and $P_{\text {sio }}=0$.

No modulation means that there is no modulation of power from any control strategy nor AVRs, i.e., $\Delta Q_{s i}=\Delta Q_{s j}=\Delta P_{s i}=0$. Furthermore, $Q_{s i}=Q_{s i o}, Q_{s j}=Q_{s j o}$ and $P_{s i}=P_{s i o}$.

\subsection{Comparison of VSC-HVdc Models. Active Power Modulation and AVR}

The injection model is compared with ABB's model in order to know how well the injection model represents the VSC-HVdc in the static and dynamic behavior of the power system. The active power is modulated by using the CLF-based signal.

\section{System loading 1 - Disturbance 1.}

The simulation results in Figure 5.3 show that the dynamic behavior of the system when using the injection model is practically the same as the result obtained with ABB's model.

\section{System loading 1 - Disturbance 3.}

In this case the results do not match as well as they did in the previous disturbance (see Figure 5.3). There is a somewhat higher damping when ABB's model is used. However the dynamic behavior of the system can still be considered very similar. Figure 5.4 shows the simulations results. 
5.3. COMPARISON OF VSC-HVDC MODELS. ACTIVE POWER

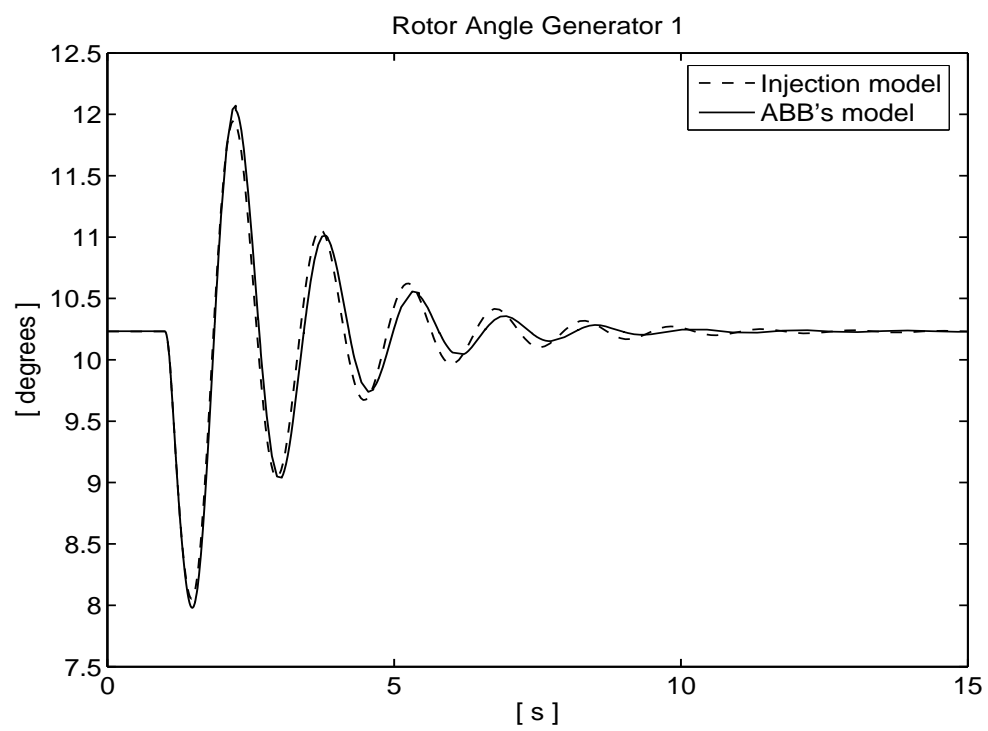

Figure 5.3: System loading 1 - Disturbance 1

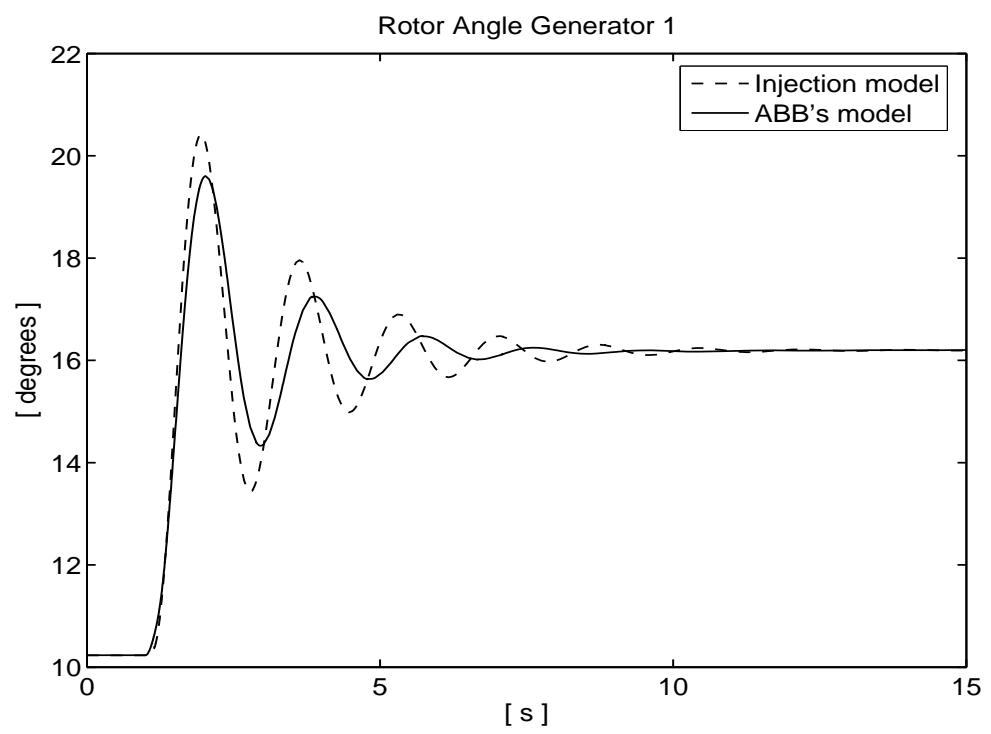

Figure 5.4: System loading 1 - Disturbance 3. 
From these two simulations it is possible to conclude that the estimation of considering the VSC-HVdc system as two ideal controllable voltage sources is sufficient for derivation and test of the control strategies for a VSC-HVdc.

\subsection{Only Active Power Modulation}

\section{System loading 3 - Disturbance 3}

When there is no modulation of power in the VSC-HVdc, the system loses synchronism in the first swing. However, when the active power is modulated by the control strategies the system is stable. Figure 5.5 shows the results.

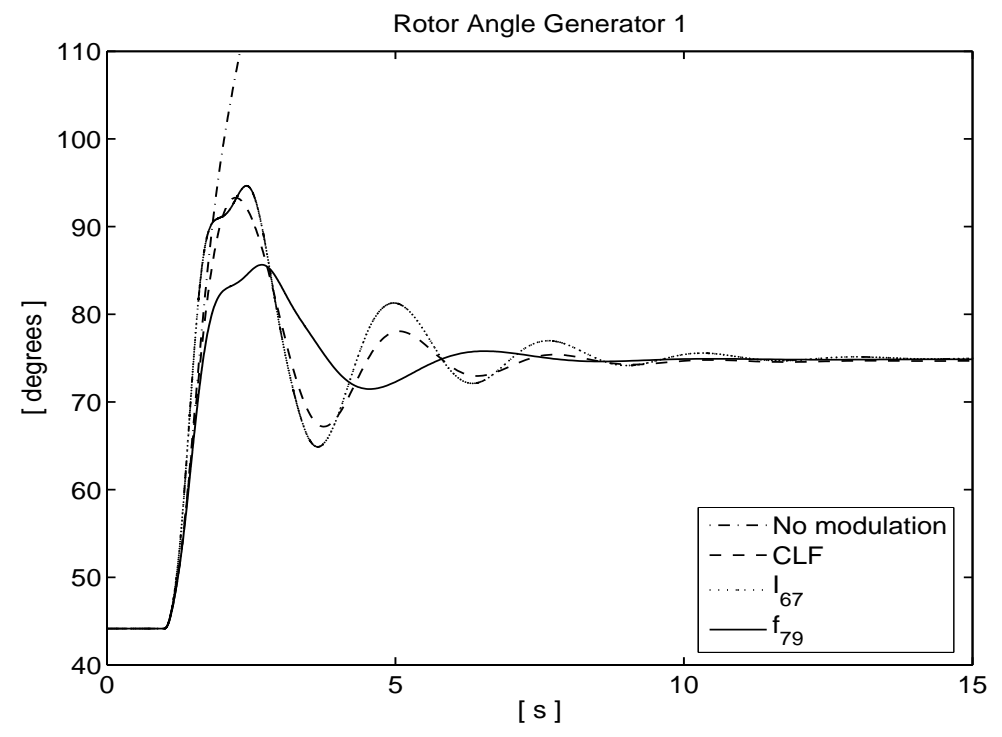

Figure 5.5: System loading 3 - Disturbance 3.

Even though CLF-based control strategy is intended for enhancing transient stability, the signal also provides damping. Modal-analysis-based signals are capable to enhance the transient stability. Besides providing damping, modal-analysis-based signal with $f_{79}$ as input signal results in lower overshoot and smaller oscillations. 


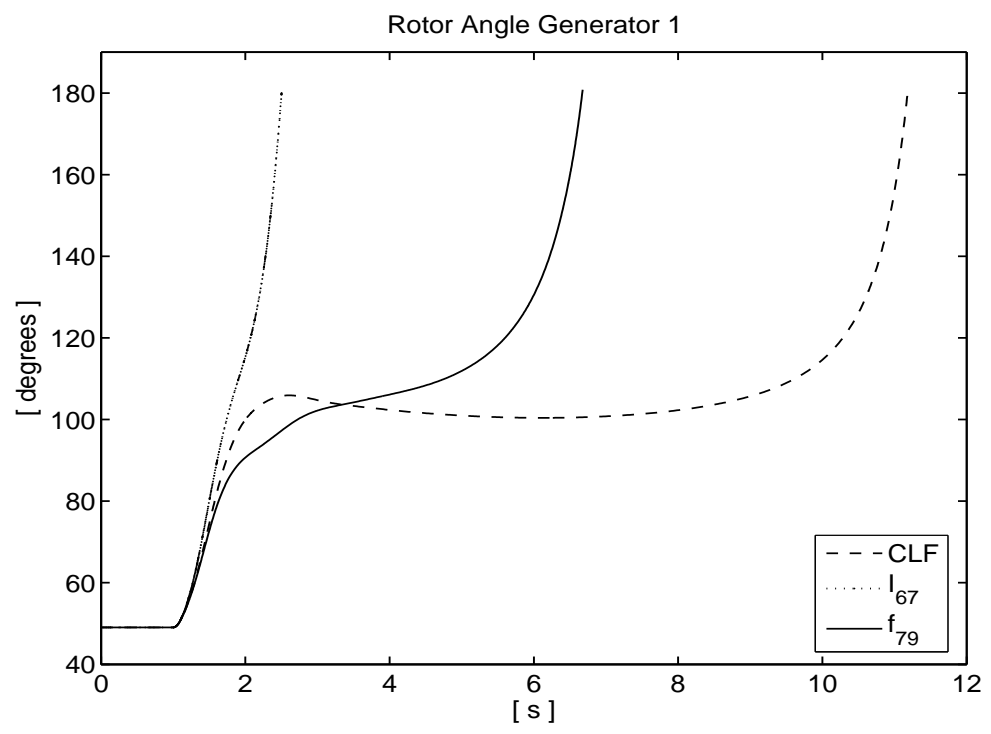

Figure 5.6: System loading 4 - Disturbance 3.

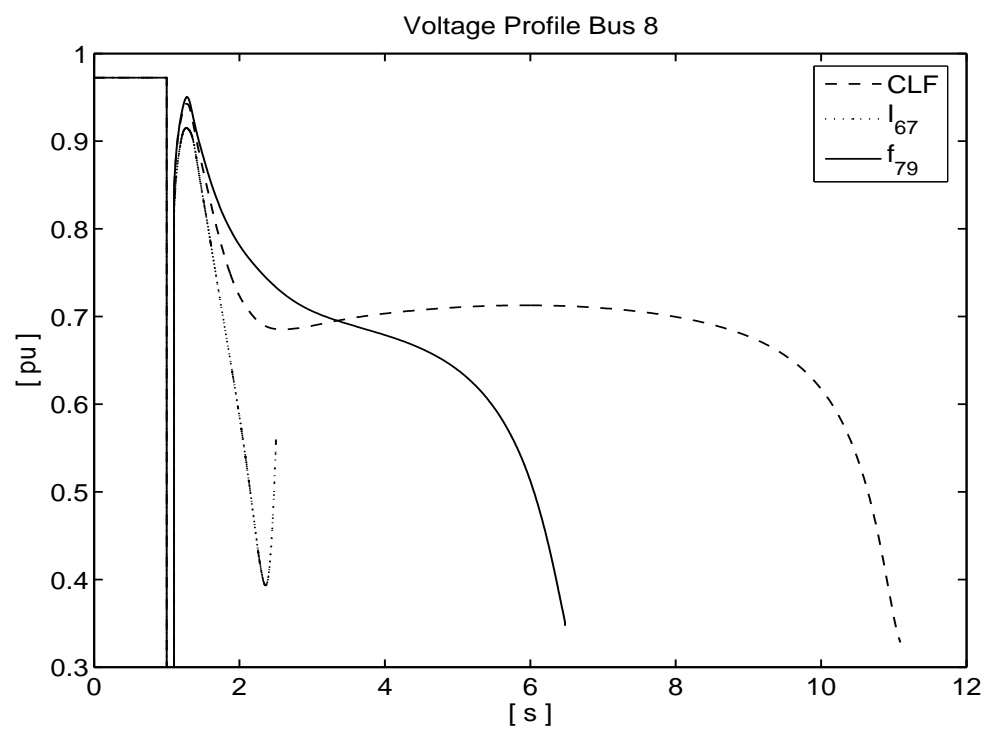

Figure 5.7: System loading 4 - Disturbance 3. 


\section{System loading 4 - Disturbance 3}

Figure 5.6 and Figure 5.7 show the simulation results. The main objective of CLF-based signal is achieved and the system is in synchronism after the first swing. However after $10 \mathrm{~s}$ the system becomes unstable mostly due to the lack of reactive power support. Concerning the modal-analysis-based signals, for both cases the system is also unstable. The power system loses synchronism even faster than the CLF-based signal. The control strategies cannot control the first swing, although $f_{79}$ keeps the system in synchronism for some more seconds compared to $I_{67}$.

It can be observed that under very heavy load conditions, the control strategies might not be enough to improve the stability of the system. Instability issues due to voltage collapse might arise in the system.

\subsubsection{Active Power Modulation and AVR}

From previous simulation it was seen that the power system also needs reactive power to increase its stability. For this reason, AVRs are enabled in the reactive power control (Figure 4.2) and will be considered in the following numerical examples.

\section{System loading 4 - Disturbance 3}

The main result is that now the system reaches the equilibrium point. With the inclusion of AVRs, the VSC-HVdc avoids the power system from falling in voltage instability. Figure 5.8 and Figure 5.9 show the simulation results.

The system is now stable with both control strategies. The increase of damping is higher with the control strategies that have the difference of frequency as input (i.e., CLF-based and modal-analysis-based strategy with $f_{79}$ as input). $f_{79}$ gives even a lower overshoot. However the post-fault steady-state voltage magnitude at bus 8 decreases to about 0.88 p.u.

\section{System loading 4 - Disturbance 1}

Figure 5.10 shows the simulation results. Voltage plot is not included since the system is marginally disturbed.

The curve of the rotor angle shows that CLF also provides damping in the system. However the increase of damping is not dramatic. It is important 


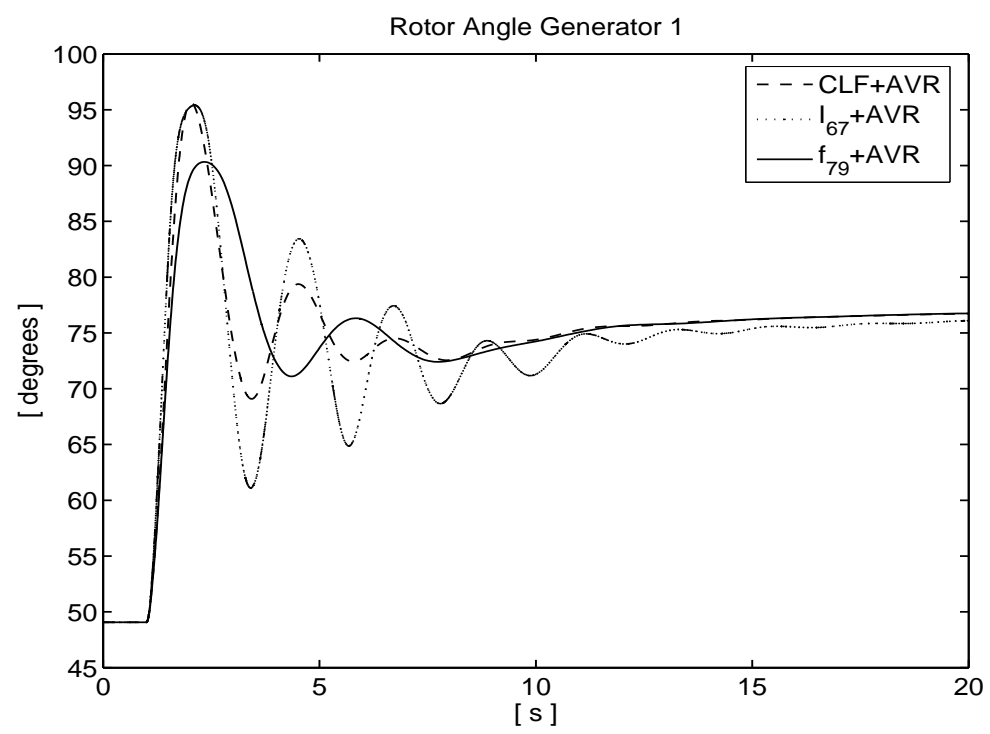

Figure 5.8: System loading 4 - Disturbance 3.

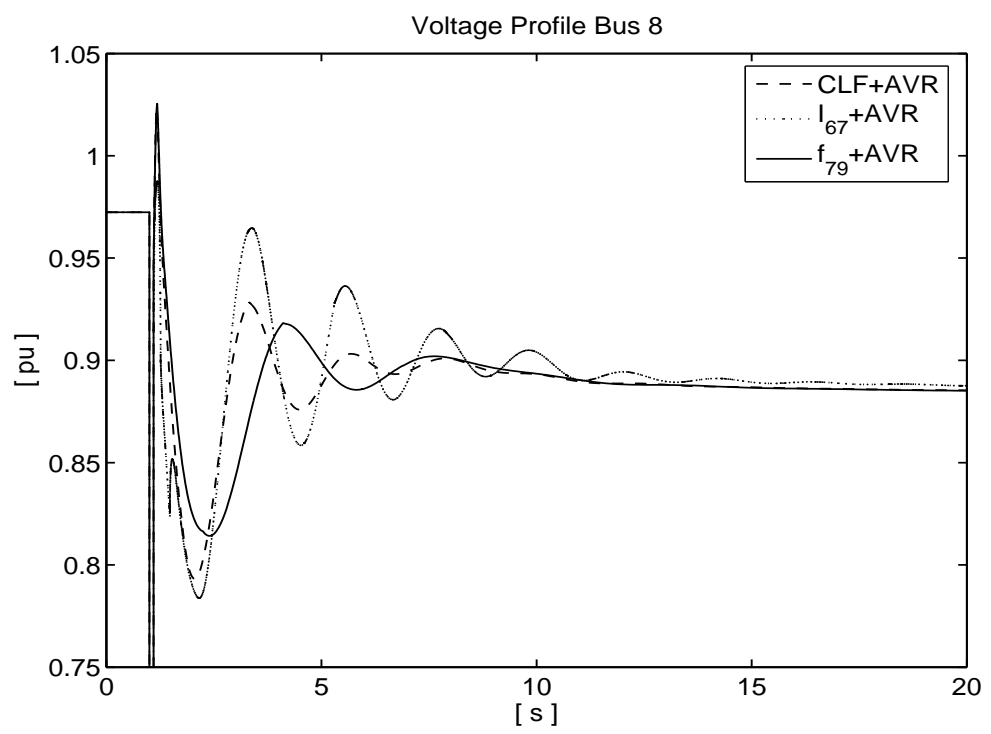

Figure 5.9: System loading 4 - Disturbance 3. 


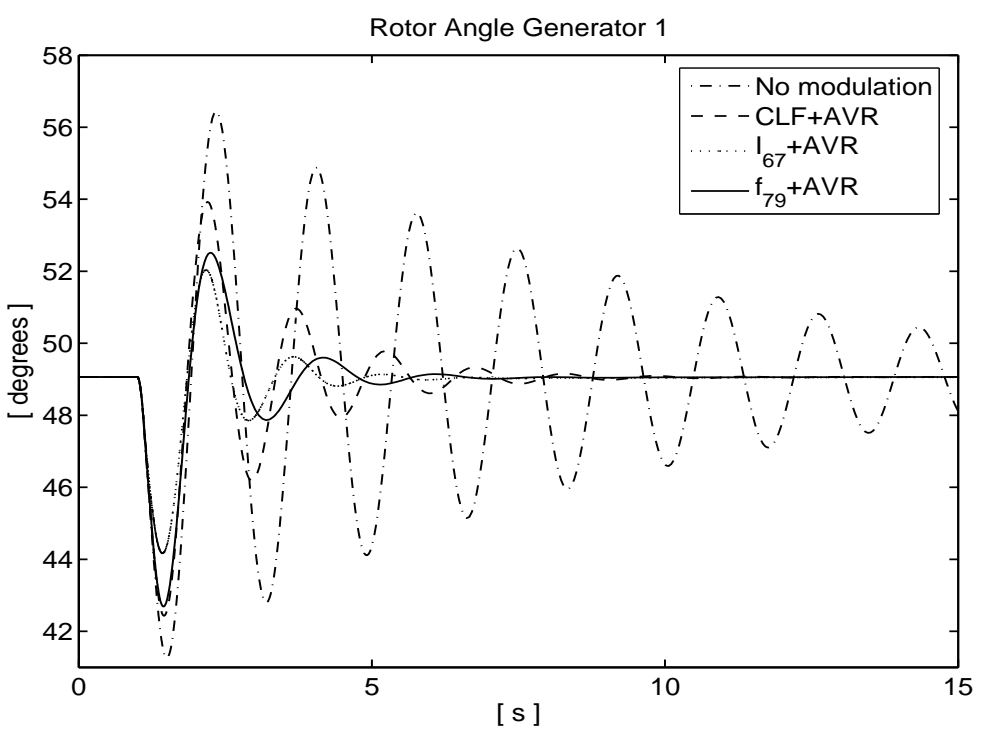

Figure 5.10: System loading 4 - Disturbance 1.

to note, nevertheless, that increase of damping is not the main objective of the CLF-based control strategy.

Both input signals in the modal-analysis-based control strategy provide very good damping in the system and the oscillations disappear practically at the same time in both curves.

\section{System loading 1 - Disturbance 4}

In this case the generators do not run out of phase during the first swing. However the lack of damping makes the system unstable when there is no modulation. Figure 5.11 - Figure 5.13 show the simulation results.

This example shows again the effectiveness of both control strategies providing damping and keeping the power system in synchronism when a substantial large disturbance occurs in the system. Concerning each control strategy, there is a noticeable difference between the damping provided for modal-analysis-based signals and the CLF-based signal. Figure 5.12 zooms the first $15 \mathrm{~s}$. After $4 \mathrm{~s}$ the oscillations disappear when modal-analysis-based control strategies are used, meanwhile CLF still registers oscillations (around $20^{\circ}$ peak to peak value). 


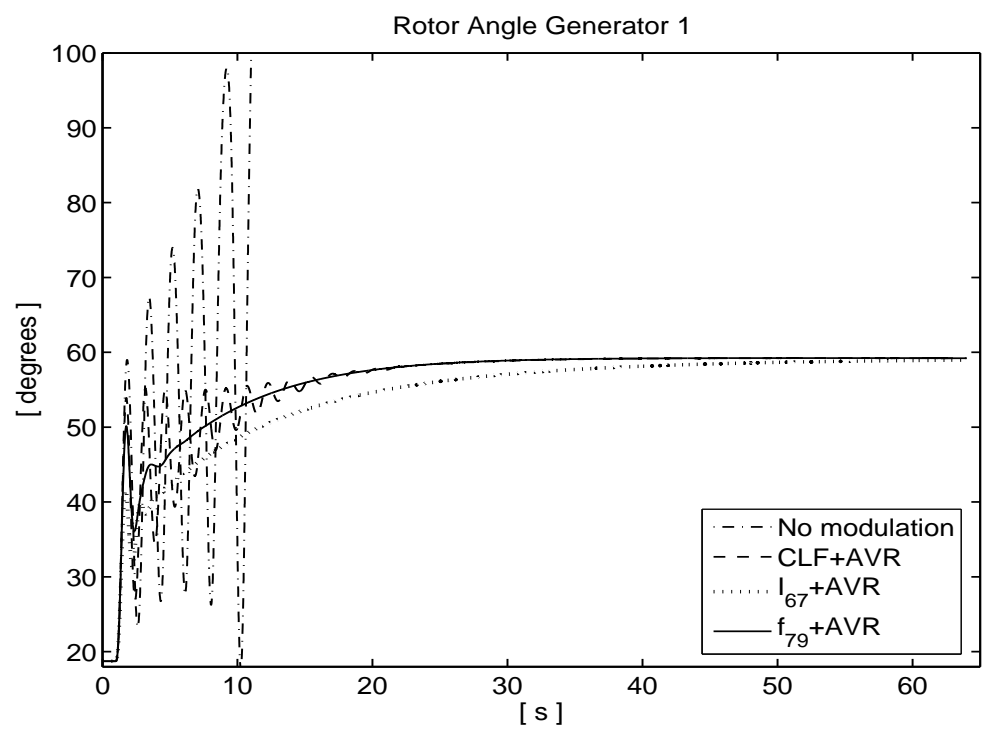

Figure 5.11: System loading 1 - Disturbance 4.

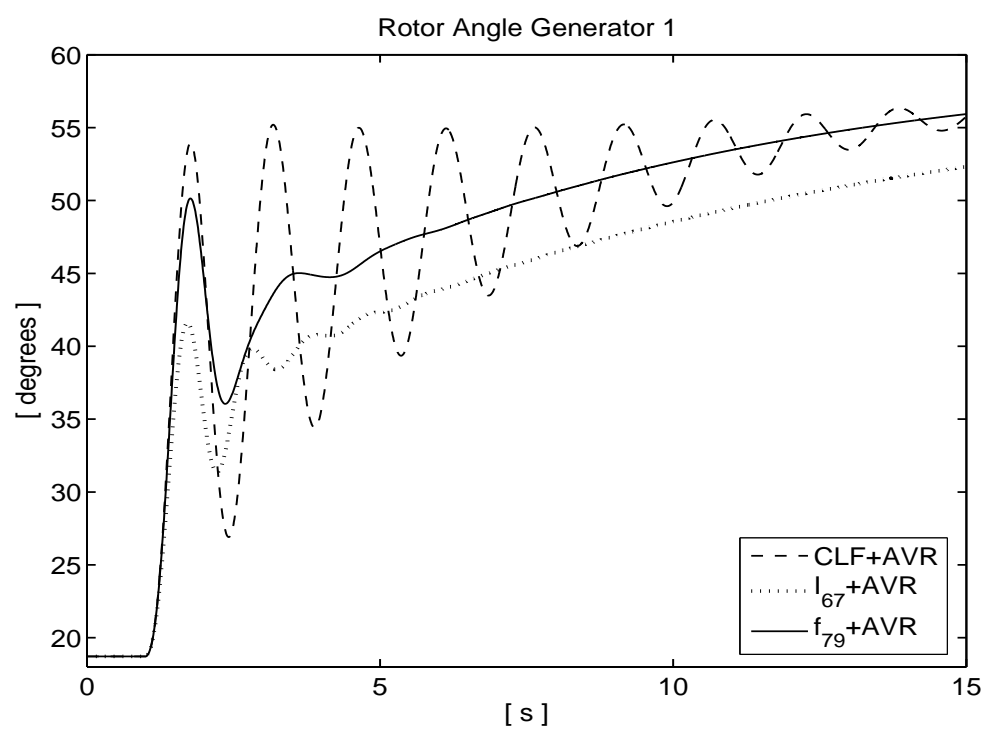

Figure 5.12: System loading 1 - Disturbance 4. 


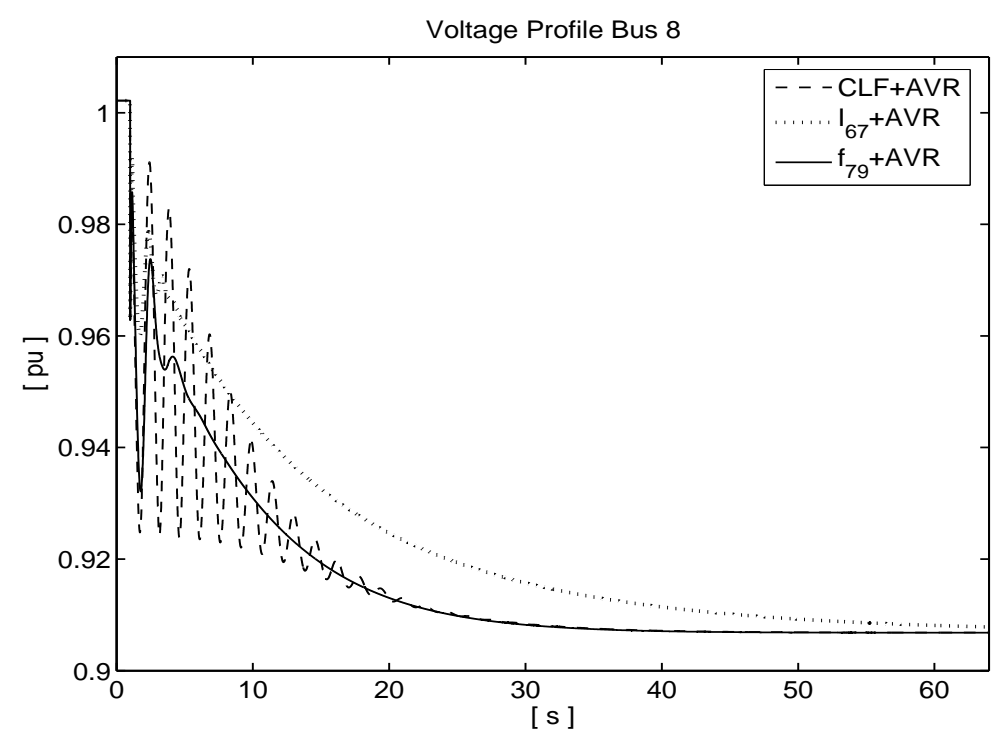

Figure 5.13: System loading 1 - Disturbance 4.

Figure 5.13 shows that the post-fault steady-state voltage magnitude at bus 8 is low (close to 0.9 p.u.). However the operator of the transmission system has more time available to perform some counteractions. The equilibrium point is reached much faster with CLF and $f_{79}$ than with $I_{67}$.

\subsection{Combination of CLF-Based Control Strategy and Modal-Analysis-Based Control Strategy}

\section{System loading 4 - Disturbance 3}

So far all the numerical examples have considered either the CLF-based control strategy or the modal-analysis-based control strategy. Both control strategies have shown their effectiveness improving the transient stability. Furthermore, they have notably provided damping in the system. One question that might arise is what the dynamic response would be if we use both control strategies (CLF and modal analysis) at the same time for modulating the active power. The objective of the following simulations is to observe if there is a positive or negative impact on the stability and damping of the 
power system when both control strategies are added. The simulation considers two cases. 1) CLF-based control strategy and modal-analysis-based control with $I_{67}$ as input signal; and 2) CLF-based control strategy and modal-analysis-based control with $f_{79}$ as input signal.

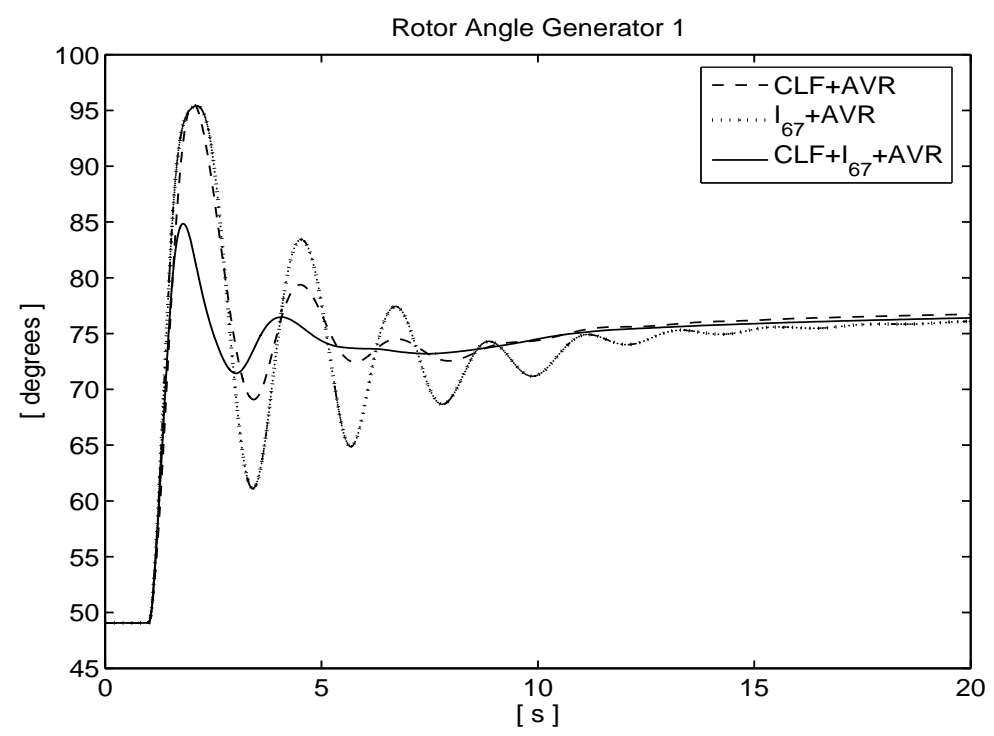

Figure 5.14: System loading 4 - Disturbance 3.

Figure 5.14 and Figure 5.15 display the simulation results. Only the curves of the rotor angle are presented. The post-fault voltage magnitude at bus 8 is the same as shown in Figure 5.9.

Both combinations impact positively the transient stability and the damping of the system. The main benefit is seen in the first swing, where the overshoot is noticeable lower than when the control strategies are individually used. The damping in the system is improved, but in a minor level. The combination CLF and $f_{79}$ gives better results than the combination CLF and $I_{67}$

\subsection{Reactive Power Control}

The major function of the modulation of reactive power is to provide voltage support at the buses where the VSC-HVdc is connected (AVRs). However, 


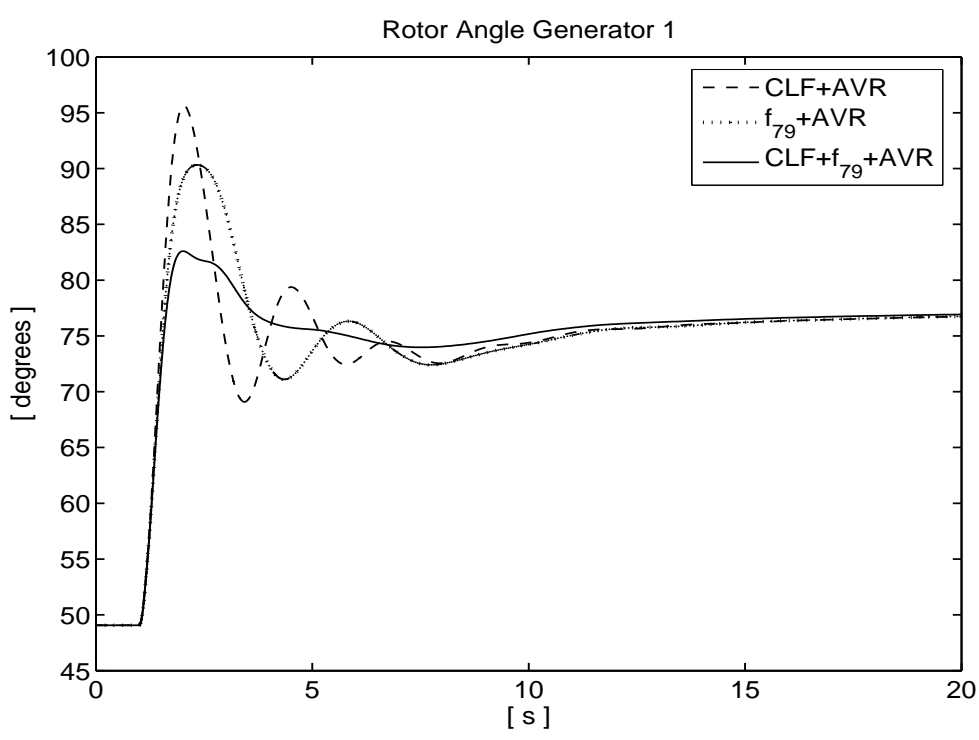

Figure 5.15: System loading 4 - Disturbance 3.

the converter stations themselves of this type of systems can also be seen as a pair of STATCOMs when there is no possibility to transfer active power through the dc-link. This condition can be exploited for providing damping in the system. It is assumed that the converters are available for operation and that the dc-cable has its own circuit breakers. The dc-cable does not depend on the ac circuit breakers.

\section{System loading 2 - Disturbance 6}

In the following numerical examples, modal-analysis-based control strategy is only considered in the converter station connected to bus 7, i.e., $K_{q j}=0$ in Figure 4.2. The reason for using only one converter is that when the control strategy is considered in each converter, one of them counteracts the effect of the other. This interaction results in a very little increase of damping in the system.

Figure 5.16 and Figure 5.17 show the simulation results with no supplementary control, i.e. $K_{q i}=K_{q j}=0$ and with a supplementary control in the first converter, i.e., $K_{q i} \neq 0$, respectively. As shown in the figures, due to this disturbance and with no supplementary control, an unstable inter- 


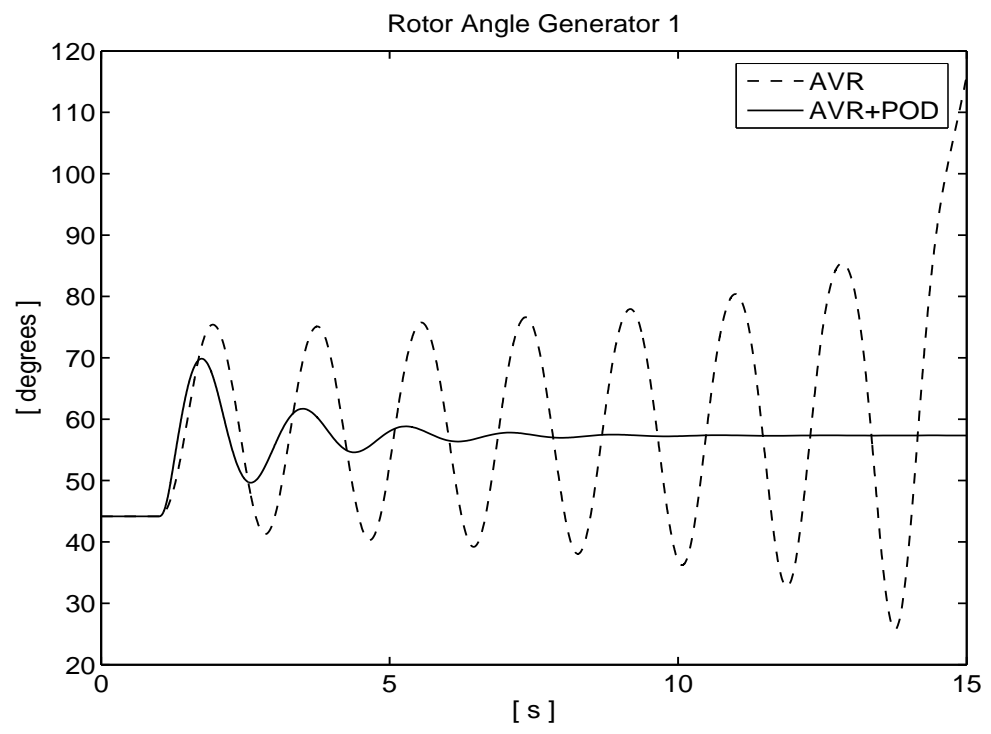

Figure 5.16: System loading 2 - Disturbance 6

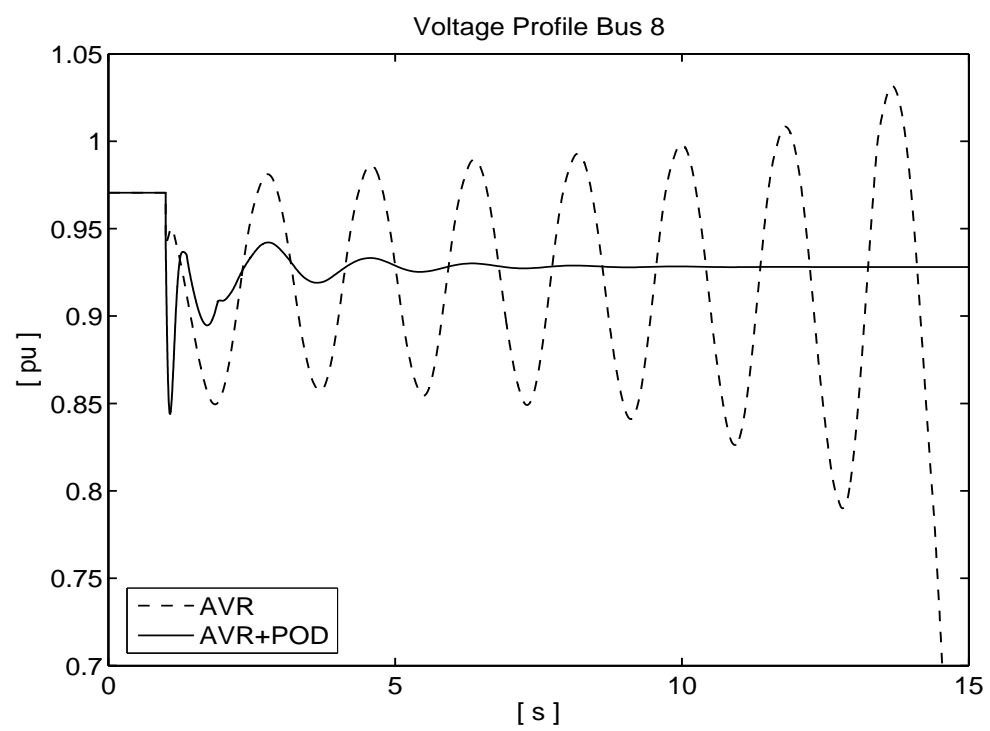

Figure 5.17: System loading 2 - Disturbance 6 
area mode is excited in the system. However, by applying a supplementary control to the reactive power modulation of the first converter, this mode becomes stable and well-damped.

\subsection{Power Flow Control}

The overall control has an additional active power input whose function is to control the power flow on the parallel ac lines.

Let us consider the results obtained for loading system 4 - disturbance 3 in section 5.4.1. In that example, the post-fault voltage at bus 8 resulted in a low value (less than 0.9 p.u.; see Figure 5.9). The objective is to raise the voltage at bus 8 to 0.95 p.u. by controlling the power flow through the ac line. This objective can be achieved by manually (and stepwise) changing the set value of the transmitted power $\left(P_{\text {sio }}\right)$ or by modulating $\Delta P_{L F}$ with the input signal $\left(U_{8}^{\text {des }}-U_{8}\right)$. Figure 5.18 shows the simulation results when the power flow is changed manually. The schedule of the stepwise order is:

$$
\text { Power control } \begin{cases}P_{\text {ref }}=P_{\text {sio }}=1.95 \text { p.u. } & \text { at } t=30 \mathrm{~s} \\ P_{\text {ref }}=P_{\text {sio }}=2.30 \text { p.u. } & \text { at } t=50 \mathrm{~s} \\ P_{\text {ref }}=P_{\text {sio }}=2.65 \text { p.u. } & \text { at } t=70 \mathrm{~s}\end{cases}
$$

Figure 5.19 shows the simulation results of the proposed modulation which is also activated at $t=30 \mathrm{~s}$, and $U_{8}^{\text {des }}=0.95$ p.u.

As shown in both cases (manually changed and modulation), by increasing the transmitted power $\left(P_{s i}\right)$ through the VSC-HVdc, the reactive power losses in the ac lines decrease and thereby less reactive power compensation by the VSC-HVdc is needed. Therefore, the injected reactive power by VSC-HVdc $\left(-Q_{s i}\right)$ decreases when the transmitted power is increasing. This results in raising the voltage at bus 8 , and also in keeping the converter apparent power $\left(S_{i}=\sqrt{P_{s i}^{2}+Q_{s i}^{2}}\right)$ below its limit, i.e. $S_{n}=3$ p.u. It is, of course, smoother and a better solution the modulation of the active power.

Since there is more capacity available in the converters to generate reactive power, the voltage support at bus 7 and bus 9 is not affected.

\section{System loading 4 - Disturbance 2}

Let us consider the results from power flow (previous numerical example) where the voltage at bus 8 was raised to $0.95 \mathrm{p}$.u. Once the voltage at 

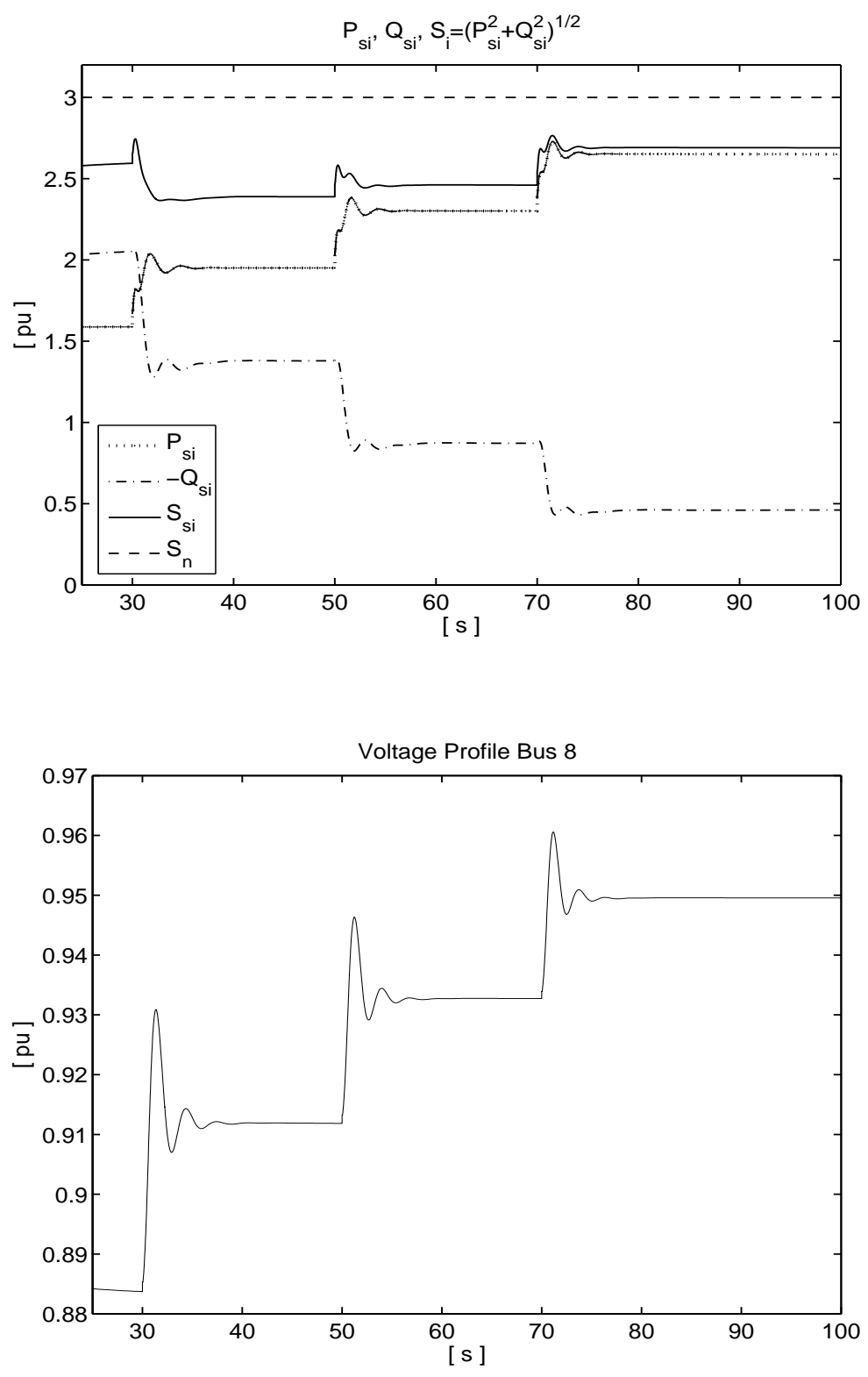

Figure 5.18: System response to manual change of $P_{\text {sio }}$. 

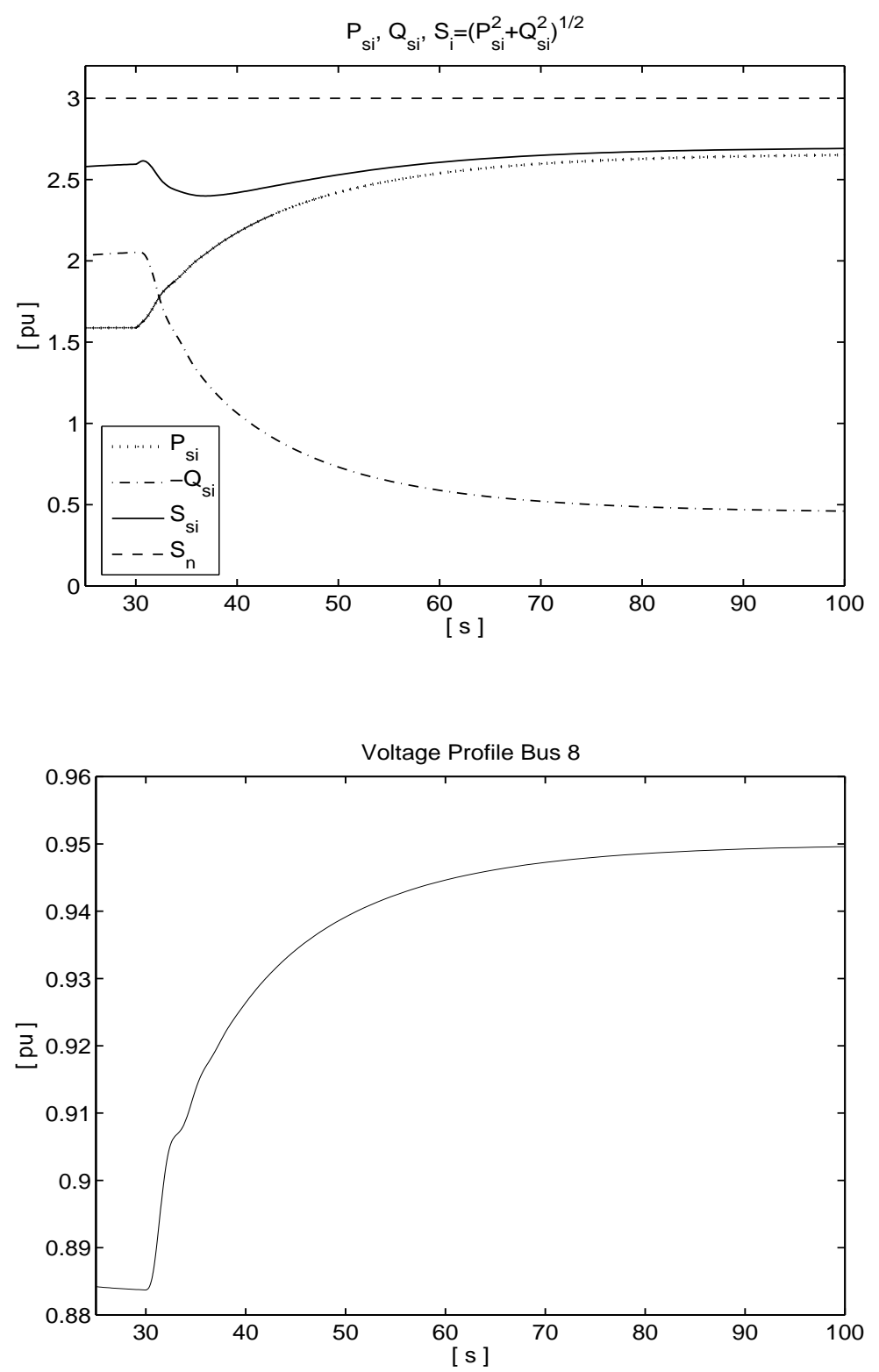

Figure 5.19: System response to modulation of $\Delta P_{L F}$. 
bus 8 has reached the reference value ( 0.95 p.u.), the reference value of the power injected into the VSC-HVdc is changed and set to the new value of transfer of power after the power flow modulation, that is, $P_{\text {sio }}$ is changed from $160 \mathrm{MW}$ to $265 \mathrm{MW}$ (see $P_{s i}$ in Figure 5.19). That means that the VSC-HVdc is switched back to the active power control mode. Under this operating conditions, a second disturbance is applied in the system. When $t=120 \mathrm{~s}$ disturbance 2 is connected. The simulation results in Figure 5.20 and Figure 5.21 show that the system is stable. The converter at bus 7 station reaches the maximum capacity since the margin for modulation of power is much lower now due to the increase of power flow. However, the small margin for modulation of power is enough to keep the system in synchronism. When this second disturbance is considered under the post-fault conditions in the numerical example system loading 4 - disturbance 3 in section 5.4.1 (Figure 5.8 and Figure 5.9) the system becomes unstable. Figure 5.22 and Figure 5.23 show the simulation results. The curves are plotted from $115 \mathrm{~s}$.

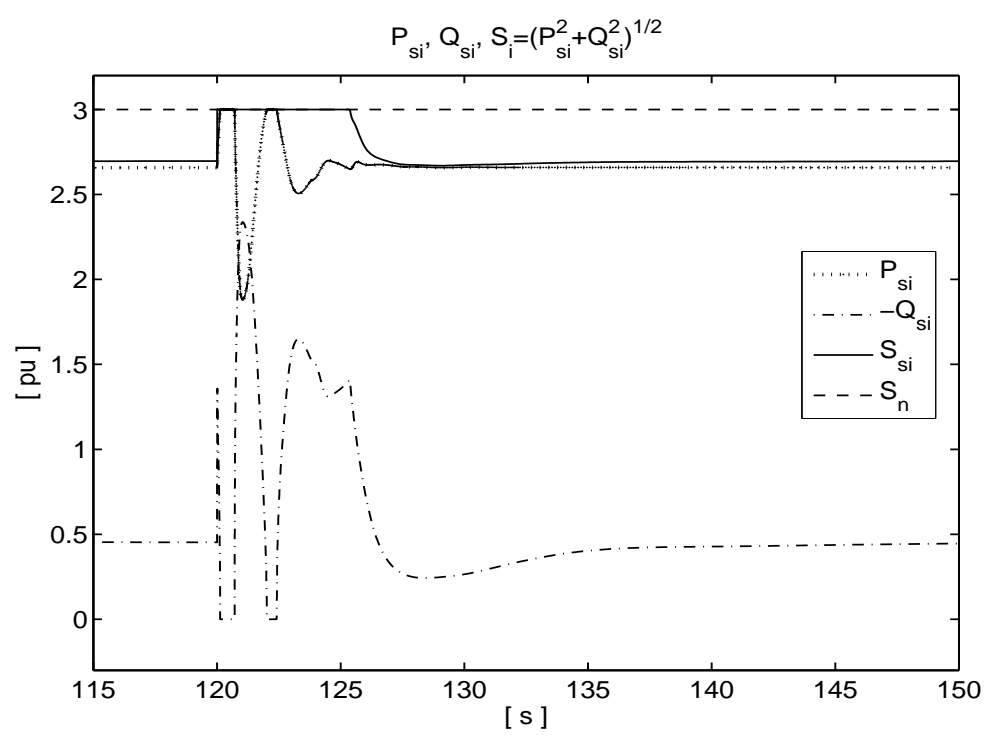

Figure 5.20: System response to disturbance 2 


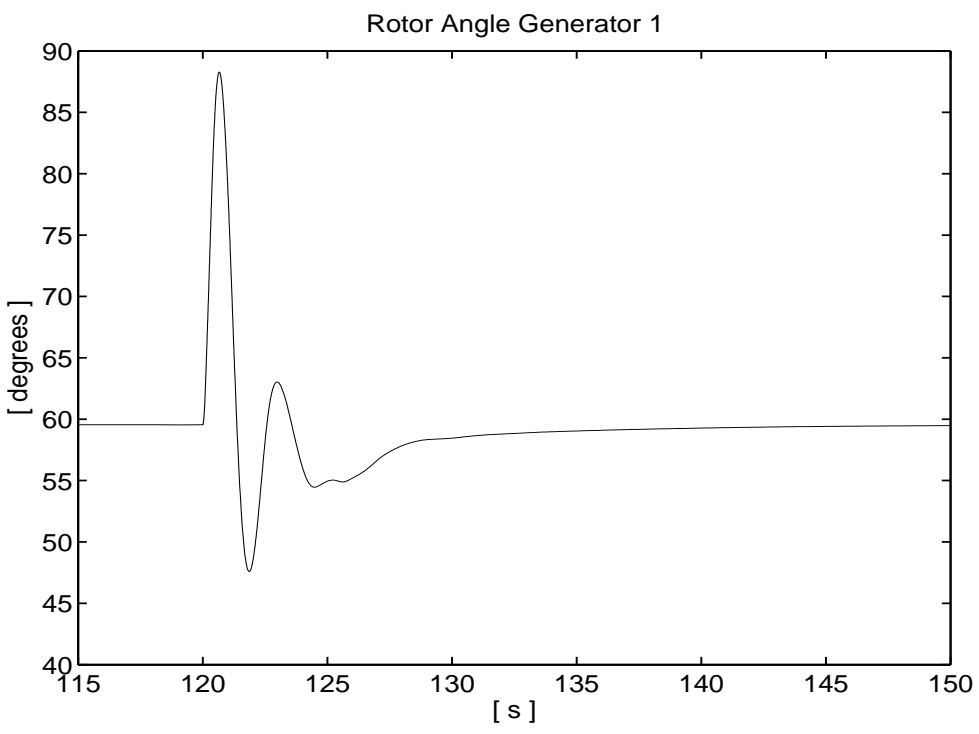

Figure 5.21: System loading 4 - Disturbance 2.

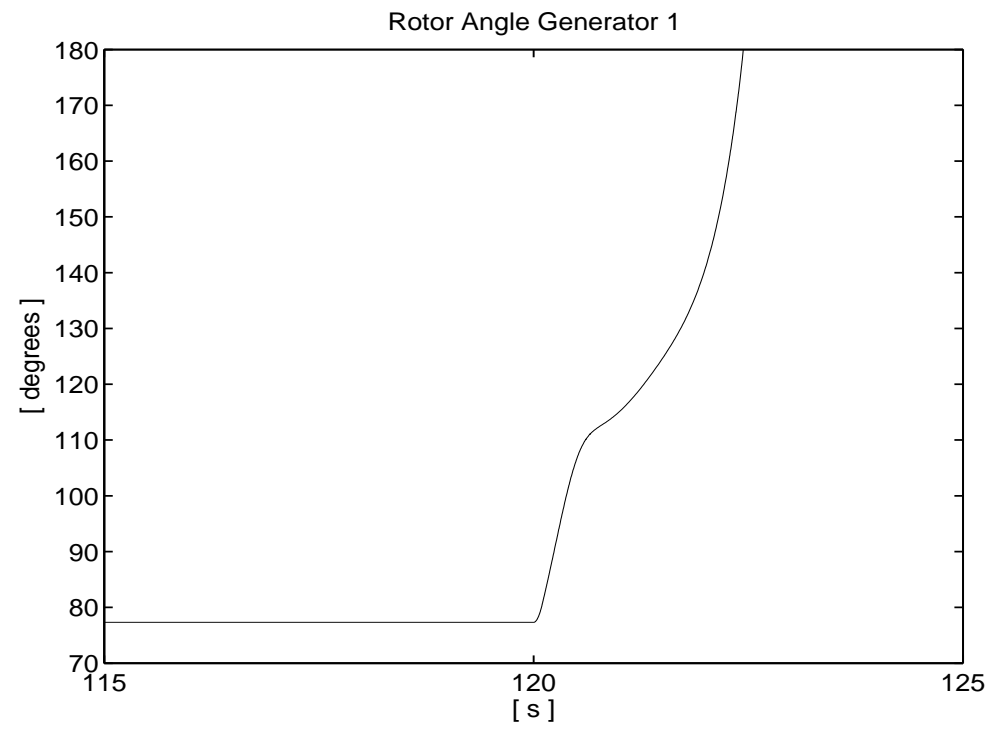

Figure 5.22: System loading 4 - Disturbance 2. 


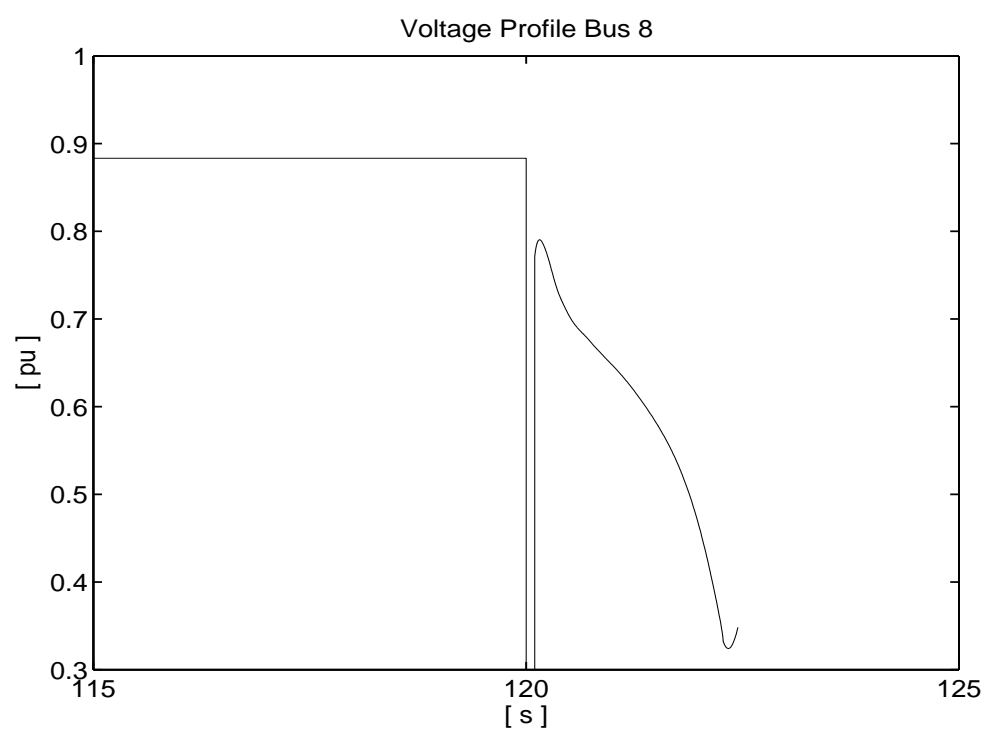

Figure 5.23: System loading 4 - Disturbance 2 



\section{Chapter 6}

\section{Conclusions and Future Work}

\subsection{Conclusions}

The thesis has analyzed the dynamic behavior of a power system when the control of a VSC-HVdc uses different derived control strategies to improve the stability and increase power oscillation damping the system. The theories that supported the derivation of the control strategies for transient stability and damping were based on two different theoretical frames Nonlinear theory and Linear theory. These two different approaches were tested in the power system individually and also combined. Voltage stability was achieved by using an Automatic Voltage Regulator (AVR) in each converter station. Each AVR tried to keep the voltage where the VSC-HVdc was connected as close as possible to the reference value. The following conclusions can be drawn:

\section{VSC-HVdc}

- It has been shown that VSC-HVdcs with a suitable control scheme can offer an alternative means to enhance transient stability, to improve power oscillations damping, and to provide voltage support, since they have the ability to rapidly control the transmitted active power, and also to independently exchange reactive power with transmissions systems.

- Based on a simplified power system model and injection model, an energy function for a power system including VSC-HVdcs has been proposed (equation (4.2)). 
2. CLF-based control strategy

- Using the proposed energy function, equation (4.2), a CLF-based control strategy for modulation of the active power of a VSCHVdc has been derived to enhance transient stability and damping. However, this control strategy has been applied to a detailed power system model with successful results. It has been shown that the proposed CLF-based control strategy has been effective to enhance transient stability (Figure 5.5). The strategy has also increased damping, but in a minor scale (Figure 5.10 and Figure 5.12)

3. Modal-analysis-based control strategy

- Two different signals were used as inputs in the modal-analysis based control strategy: $I_{67}$ and $f_{79}$. The signals remarkably improved the power oscillation damping in the system (Figure 5.10 and Figure 5.12). Both signals also showed its capability of improving the transient stability (Figure 5.5). The modal-analysisbased active power control is more effective and robust with the input signal $\Delta f=f_{79}$ than with the signal $I_{67}$. The reason might be that $\Delta f=f_{79}$ contains global information, but $I_{67}$ only contains local information.

4. Modulation of reactive power

- Both control strategies CLF-based and modal-analysis-based have shown to be very effective enhancing the transient stability of the system and providing damping. However, when the power system is heavily loaded, it has been shown that only modulation of active power is not sufficient to stabilize the system (Figure 5.6). Therefore, the modulation of the reactive power of the VSC-HVdc must be considered. Figure 5.8 and Figure 5.9 clearly show that the reactive power control has a significant contribution on system stability and damping.

- Since the VSC-HVdc can be seen as two STATCOMs when there is no transfer of dc-power, a supplementary control was derived (based on modal analysis) to improve damping in the system. 
It has been shown that the proposed control strategy effectively increased the damping in the system (Figure 5.16).

5. Combination of CLF-Based Control Strategy and Modal-AnalysisBased Control Strategy

- The Combination of CLF-based and modal-analysis-based control strategies resulted in slightly better results than the cases when they were considered individually. The main impact of this combination was on the first swing. The peak value of the first oscillation was notably reduced (Figure 5.14 and Figure 5.15). Damping was also improved, but in a lower level. The combination of CLF and the modal-analysis-based signal with $f_{79}$ as input showed to be more effective than the combination CLF and $I_{67}$.

6. Power flow control

- The VSC-HVdc provides an excellent way to perform power flow control in the system. The control can be done either manually (and stepwise) by setting a new reference value of power $P_{\text {sio }}$ or by modulation defining an objective function. By modulating the active power, the system has a smoother response (Figure 5.19).

7. Multichoice control scheme

- The simulation results show that a VSC-HVdc with a multichoice control scheme (e.g., Figure 5.2) has the ability to optimize utilization of the existing transmission systems, and at the same time to keep high system reliability and security

\subsection{Future Work}

The project has derived different control strategies for enhancing stability and improving damping of a system. It has also proposed a control scheme to optimize the benefits of the VSC-HVdc. Based on those results achieved, it would be of interest to study in deeper detail the following subjects:

- Analysis of the impact of a VSC-HVdc on much more complex power system when it is connected in the transmission network. The analysis 
would include an examination of the changes in the oscillation modes; identification and control of the most interesting oscillatory modes; and derivation of control strategies to increase the stability of the system. It would also be interesting to explore the possibility to control multiple oscillations.

- Use of remote information based on PMUs. The numerical examples presented in Chapter 5 read the signals directly from the buses (frequency). PMUs might be considered to be installed either in generators (rotor angle), or network buses (voltage angle) or in both. The analysis of using remote information would be compared with local information regarding both transient stability and increase of power oscillation damping. The number of PMUs installed in the system would be determined by Modal Analysis theory or SIngle Machine Equivalent -SIME-.

- Enhancement of transient stability and reduction of electromechanical oscillations are better achieved with a combination of modulation of $\mathrm{P}$ and $\mathrm{Q}$. The project has not derived a strategy to define an optimum value for $\mathrm{P}$ and $\mathrm{Q}$ under fault conditions. Priority was given to $\mathrm{P}$ and according to the rating of the converters, $\mathrm{Q}$ was determined (component of Q defined from AVR). Future work could define a control that would be able to find the optimum relation of how much $\mathrm{P}$ and how much Q must be modulated when a disturbance occurs in the system. This type of control would become an adaptive control.

- Further analysis of voltage stability. Under normal operating conditions, the use of the reactive power in the VSC-HVdc is low. However in order to enhance the voltage in the grid a strategy could be defined so that the power flow along the HVdc and parallel ac lines is changed and more capacitive for modulation of $\mathrm{Q}$ is available. Coordination with close by generators could also be included so that the production/generation of $\mathrm{Q}$ is optimized.

- Analysis of the interaction between the converter stations when they provide damping in the system. In this project only one converter was used to provide damping, since when both VSC provided damping there were ceratin interaction between them that resulted in almost zero increase of damping. 


\section{Bibliography}

[1] Imperial College London, Department of Electrical and Electronic Engineering. Project: "Robust Damping of Inter-Area Oscillations in Power Systems Using FACTS Controllers". http://www3.imperial.ac.uk/controlandpower

[2] M. Venkatasubramanian, "Analyzing Blackout Events: Experience from the Major Western Blackouts in 1996," Power System Research Center, Aug. 20, 2003

[3] H. K. Clark, "New Challenge: Voltage Stability," IEEE Power Engineering Review, vol. 10, pp 33-37, 1990.

[4] K. R. Padiyar and S. S. Rao, "Dynamic Analysis of Small Signal Voltage Instability Decoupled from Angle Instability," Electrical Power 83 Energy Systems, vol. 18, no. 7, pp 445-452, 1996.

[5] U.S.-Canada Power System Outage Task Force, "Final Report on the August 14th, 2003 Blackout in the United States and Canada: Causes and Recommendations", April 2004.

[6] C. D. Vournas, G. A. Manos, J. Kabouris and T. Van Cutsem, "Analysis of Voltage Instability Incident in the Greek power System," Power Engineering Society Winter Meeting, vol. 2, pp. 1483-1488, Jan. 2000

[7] L. Zhang, L. Harnefors, P. Rey, "Power System Reliability and Transfer Capability Improvement by VSC-HVdc," CIGRÉ Regional Meeting , June, 2007.

[8] S. G. Johansson, G. Asplund, E. Jansson, R. Rudervall, "Power System Stability Benefits with VSC dc-Transmission Systems," CIGRÉ, Paris, France, 2004 
[9] A. Petersson, A. Edris, "Dynamic Performance of the Eagle Pass Backto-Back HVDC Light Tie,", AC-DC Power Transmission, Nov., 2001

[10] Z. Huang, B. T. Ooi, L. A. Dessaint, F. D. Galiana, "Exploiting Voltage Support of Voltage Source HVdc," IEE Proceeding online no. 2003099, Jan., 2003

[11] P. Bresesti, W. L. Kling, R. L. Hendriks,R. Vailati,"HVdc Connections of Offshore Wind Farms to the Transmission System (Invited paper)," IEEE Transactions on Energy Conversion, vol. 22, no. 1, pp 37-43, Mar. 2007.

[12] X. Yao, H. Sui, Z. Xing,"The Study of VSC-HVdc Transmission System for Offshore Wind Power Farm," International Conference on Electrical Machines and Systems, Seoul, Korea, Oct., 2007.

[13] K. Habur and D. OĹeans, "FACTS-flexible alternating current transmission systems: for cost effective and reliable transmission of electrical energy," available at www.worldbank.org/html/fpd/em/transmission/facts_siemens.pdf, Aug. 15, 2004.

[14] D. Kirschen and G. Strbac, "Why Investments Do Not Prevent Blackouts," The Electricity Journal, Vol. 17, Issue 2, pp. 29-36, March 2004.

[15] L. Kirschner, D. Retzmann, G. Thumm, "Benefits of FACTS for Power System Enhancement," IEEE/PES Transmission and Distribution Conference 83 Exhibition: Asia and Pacific, Dalian, China, 2005.

[16] R. Grünbaum, B. Halvarsson, A. Wilk-Wilczynski, "FACTS and HVDC Light for Power System Interconnections," Power Delivery, Madrid, Spain, 1999.

[17] —. (2007, Nov). ABB HVDC hompepage. [Online]. Available: http://www.abb.com/hvdc

[18] S. Cole, D. Van Hertmen, I. Pardon, R. Belmans, "Randstad HVDC," Department of Electrical Engineering, KULeuven, Aug. 8, 2006 .

[19] —. (2007, Nov). ABB HVDC hompepage. [Online], "HVDC \& SVC Light- Reference List". Available: http://www.abb.com/hvdc 
[20] M. H. Haque, "Improvement of First Swing Stability Limit by Utilizing Full Benefit of Shunt FACTS Devices," IEEE Transactions on Power Systems, vol. 19, no. 4, Nov. 2004.

[21] M. H. Haque, "Application of energy function to assess the firstswing stability of a power system with a SVC," IEE Proceedings Generation, Tranmission and Distribution, Vol. 152, No. 6, Nov. 2005.

[22] M. Ghandhari, G. Andersson and I. A. Hiskens, "Control Lyapunov Functions for Controllable Series Devices", IEEE Transactions on Power Systems, vol. 16, no. 4, Nov. 2001.

[23] M. Ghandhari, "Application of Control Lyapunov Functions to Static Var Compensator," IEEE Proceedings Conference on Control Applications, Sept. 18-22, Glasgow, Scotland, 2002.

[24] V. Azbe, U. Gabrijel, D. Povh and R. Mihalic, "Energy Function of a General Multimachine System With a Unified Power Flow Controller," IEEE Transactions on Power Systems, vol. 20, no. 3, Aug. 2005.

[25] E. V. Larsen, J. J. Sanchez-Gasca and J. H. Chow, "Concepts for Design of Facts Controllers to Damp Power Swings," IEEE Transactions on Power Systems, vol. 10, no. 2, May 1995.

[26] L. E. Jones and G. Andersson, "Selecting Robust Input Signals for HVdc Damping Controllers," IEE AC and DC Power Transmission, Conference, no. 423, April 1996.

[27] F. A. Rahman and B. T. Ooi, "VSC-HVdc Station with SSSC Characteristics," IEEE Transactions on Power Electronics, vol. 19, no. 4, July 2004 .

[28] W. Lu and B. T. Ooi, "Simultaneous Inter-area Decoupling and Local Area Damping by Voltage-Source HVdc," IEEE Power Engineering Society Winter Meeting, vol. 3, pp. 1079-1084, Feb 2001.

[29] S. G. Johansson, L. Carlsson and G. Russberg, "Explore the Power of HVdc Light -a Web Based System Interaction Tutorial," IEEE Power Systems Conference and Exposition, vol. 2, Oct. 10-13, 2004. 
[30] M. P. Bahrman and B. K. Johnson. "The ABCs of HVDC Transmission Technologies", IEEE Power \&f Energy, Vol. 5, No. 2, pp. 34-35 and 44, Mar./Apr. 2007.

[31] G. Asplund, "Sustainable Energy Systems with HVDC Transmission", IEEE PES General Meeting, Vol. 2, pp. 2299-2303, Jun. 2004

[32] M. Ghandhari, "Dynamic Analysis of Power System Part II," KTH, Stockholm, 2007.

[33] J. Machowski, J. W. Bialek and J. R. Bumby, "Power System Dynamics and Stability," USA: John Wiley \& Sons, 1997

[34] H. K. Khalil, "Nonlinear Systems," USA: Prentice-Hall, 2000.

[35] M. A. Pai, "Energy Function Analysis for Power System Stability," Kluwer Academic Publishers, 1989.

[36] M. Ghandhari, "Control Lyapunov Functions: A Control Strategy for Damping of Power Oscillations in Large Power Systems," Doctoral Dissertation, Royal Institute of Techonology-KTH-, Stockholm 2000.

[37] Z. Artstein, "Stabilization with relaxed controls", Nonlinear Analysis, Theory, Methods and Applications, Vol. 7, No. 11, pp. 1163-1173, 1983.

[38] E. Sontag, "A universal construction of Artstein's theorem on nonlinear stabilization", Systems and Controll Letters 13, pp. 1163-1173, 1983.

[39] V. Jurdjevic and J. P. Quinn, "Controllability and Stability", Journal of Differential Equations 28, pp. 381-389, 1978.

[40] E. Sontag, "Stability and Stabilization: Discontinuities and the Effect of Disturbances," NATO Advanced Study Institute, pp. 551-598, Montreal 1998.

[41] F. L. Pagola, I. J. Perez-Arriaga, G. C. Verghese, "On Sensitivities, Residues and Participation. Application to Oscillatory Stability Analysis and Control", IEEE Transactions on Power Systems, vol. 4, no. 1, Feb. 1989.

[42] P. Kundur, "Power System Stability and Control," New York: McGrawHill, 1994, pp. 813 
[43] N. Martins, L. T. G. Lima, "Eigenvalue and Frequency Domain Analysis of Small Signal Electromechanical Stability Problems", Eigenanalysis and Frequency Domain Methods for System Dyamic Performance, IEEE, New York, 1989

[44] M. Pavella and P.G. Murthy, "Transient Stability of Power Systems, Theory and Practice," John Wiley \& Sons, 1994.

[45] STRI, "SIMPOW - a Digital Power System Simulator," ABB Review No. 7, 1990 